Article

\title{
Novel Bis-Ammonium Salts of Pyridoxine: Synthesis and Antimicrobial Properties
}

\author{
Nikita V. Shtyrlin ${ }^{1}$, Mikhail V. Pugachev ${ }^{1}$, Sergey V. Sapozhnikov ${ }^{1}{ }^{\mathbb{B}}$, Marsel R. Garipov ${ }^{1}$, \\ Rusalia M. Vafina ${ }^{1}$, Denis Y. Grishaev ${ }^{1}$, Roman S. Pavelyev ${ }^{1}$, Renata R. Kazakova ${ }^{1}$, \\ Mariya N. Agafonova ${ }^{1}$, Alfiya G. Iksanova ${ }^{1}$, Svetlana A. Lisovskaya ${ }^{2}{ }^{\mathbb{D}}$, Marina I. Zeldi ${ }^{1}$, \\ Elena S. Krylova ${ }^{1}$, Elena V. Nikitina ${ }^{1}\left(\mathbb{D}\right.$, Alina E. Sabirova ${ }^{1} \mathbb{D}$, Airat R. Kayumov ${ }^{1}(\mathbb{D})$ and \\ Yurii G. Shtyrlin ${ }^{1, *}$ \\ 1 Kazan (Volga region) Federal University, Scientific and Educational Center of Pharmaceutics, \\ Kremlyovskaya St. 18, Kazan 420008, Russia; Nikita.Shtyrlin@kpfu.ru (N.V.S.); \\ pugachev.mihail@gmail.com (M.V.P.); sapozhnikovsergei@gmail.com (S.V.S.); \\ MarRGaripov@kpfu.ru (M.R.G.); rusalia.wafina@kpfu.ru (R.M.V.); DJGrishaev@kpfu.ru (D.Y.G.); \\ rpavelyev@gmail.com (R.S.P.); kazakova.renata@mail.ru (R.R.K.); Mariya.Agafonova@kpfu.ru (M.N.A.); \\ Alfija.Iksanova@kpfu.ru (A.G.I.); zeldimarina@gmail.com (M.I.Z.); elena_krylova.stud2015@mail.ru (E.S.K.); \\ ev-nikitina@inbox.ru (E.V.N.); alinka.zam@mail.ru (A.E.S.); Ajrat.Kajumov@kpfu.ru (A.R.K.) \\ 2 Kazan Scientific Research Institute of Epidemiology and Microbiology, Kazan 420015, Russia; \\ s_lisovskaya@mail.ru \\ * Correspondence: yurii.shtyrlin@kpfu.ru; Tel.: +7-843-233-7363
}

Academic Editor: Bogumil E. Brycki

Received: 21 August 2020; Accepted: 17 September 2020; Published: 22 September 2020

\begin{abstract}
A series of 108 novel quaternary bis-ammonium pyridoxine derivatives carrying various substituents at the quaternary nitrogen's and acetal carbon was synthesized. Thirteen compounds exhibited antibacterial and antifungal activity (minimum inhibitory concentration (MIC) $0.25-16 \mu \mathrm{g} / \mathrm{mL}$ ) comparable or superior than miramistin, benzalkonium chloride, and chlorhexidine. A strong correlation between the lipophilicity and antibacterial activity was found. The most active compounds had $\log \mathrm{P}$ values in the range of $1-3$, while compounds with $\log \mathrm{P}>6$ and $\log \mathrm{P}<0$ were almost inactive. All active compounds demonstrated cytotoxicity comparable with miramistin and chlorhexidine on HEK-293 cells and were three-fold less toxic when compared to benzalkonium chloride. The antibacterial activity of leading compound $\mathbf{5 c}_{\mathbf{1 2}}$ on biofilm-embedded Staphylococcus aureus, Staphylococcus epidermidis, Escherichia coli or Pseudomonas aeruginosa was comparable or even higher than that of the benzalkonium chloride. In vivo $\mathbf{5} \mathbf{c}_{12}$ was considerably less toxic ( $\mathrm{LD}_{50} 1705 \mathrm{mg} / \mathrm{kg}$ ) than benzalkonium chloride, miramistine, and chlorhexidine at oral administration on CD-1 mice. An aqueous solution of $5 \mathbf{c}_{\mathbf{1 2}}(0.2 \%)$ was shown to be comparable to reference drugs efficiency on the rat's skin model. The molecular target of $5 \mathbf{c}_{12}$ seems to be a cellular membrane as other quaternary ammonium salts. The obtained results make the described quaternary bis-ammonium pyridoxine derivatives promising and lead molecules in the development of the new antiseptics with a broad spectrum of antimicrobial activity.
\end{abstract}

Keywords: quaternary ammonium salts; pyridoxine; antibacterial activity; antifungal activity; cytotoxicity; biofilms; antiseptics

\section{Introduction}

The drastic worldwide rise of antimicrobial drug resistance would lead to the death of 10 million people every year by 2050, which becomes a proven and challenging problem [1,2]. In comparison with antibiotics for systemic application, antiseptics are characterized by a wider spectrum of antimicrobial 
action against bacteria, fungi, viruses, and protozoa [3,4]. Therefore, antiseptics are an important integral part of stewardship strategies for preventing and treating the surgical site and chronic open wound infections [5].

QAC (quaternary ammonium compounds) is one of the best-known group of antiseptics. The prominent representatives of this class are benzalkonium chloride, cetylpyridinium chloride, miramistin, and didecyldimethylammonium chloride [6,7]. The mechanism of the antibacterial action of QAC consists of their adsorption and penetration through the cell wall of bacteria, which is followed by an interaction with phospholipids of the cytoplasmic membrane and subsequent complete structural disorganization of the membrane and subsequent cell death [3].

Since QAC-based antiseptics could be inefficient because of acquired resistance [3,8], have high toxicity [9], lead to skin irritation [10], and demonstrate low activity against spores, simple viruses, Gram-negative bacteria, mycobacteria, and fungi [11], the development of new antiseptic molecules is strictly required. Therefore, the goal of this study was developing new drugs that would strengthen all the advantages of QAC and eliminate their disadvantages as well.

In our group, we have systematically studied chemistry and biological properties of the pyridoxine (vitamin B6) derivatives [12]. In previous works, we described a series of QAC based on pyridoxine derivatives [13-17]. Some of them possess high antibacterial activity against various Gram-positive and Gram-negative bacteria including multi-resistant clinical isolates (MICs $=0.5-16 \mu \mathrm{g} / \mathrm{mL}$ ) and low toxicity in vitro. In our patents $[18,19]$, we described the synthesis of the bis-ammonium salt $\mathbf{5} \mathbf{c}_{\mathbf{1 2}}$ with advantageous antimicrobial properties (Figure 1). These promising results encouraged us to synthesize and study an extended set of modified structural analogs of $\mathbf{5} \mathbf{c}_{\mathbf{1 2}}$, which bears various substituents at the quaternary nitrogens and acetal carbon.

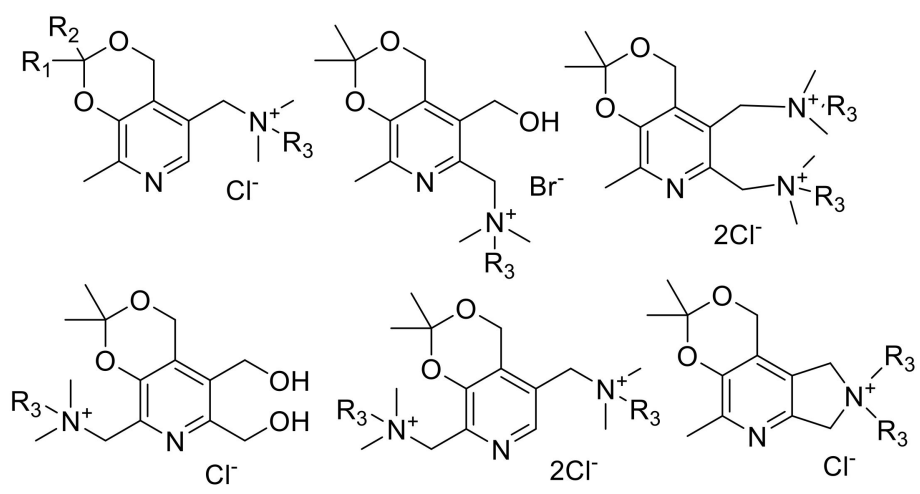<smiles>[R]C1([R])OCc2c(C[N+]([Y])(C)Cl)cnc(C[N+](C)(C)C)c2O1</smiles>

Figure 1. The structures of QAC based on pyridoxine derivatives obtained by us earlier [13-19] and compounds $\mathbf{5 a}_{\mathbf{8}}-\mathbf{5} \mathbf{r}_{18}$ synthesized in this work.

\section{Results and Discussion}

In the work [18], we described the first synthesis of compound $\mathbf{5 c}_{\mathbf{1 2}}$ in seven steps with the overall yield of only $13 \%$. Then we developed a new synthetic approach in four steps with an overall yield of $22 \%$ [19]. For the synthesis of its analogues $5 \mathbf{a}_{\mathbf{8}}-\mathbf{5} \mathbf{r}_{\mathbf{1 8}}$, we used the same approach with some modifications (Scheme 1). An approach includes the initial preparation of six-membered acetals $\mathbf{2 a}-\mathbf{r}$ with different substituents at acetal carbon [20,21]. At the second stage, in reaction of $\mathbf{2} \mathbf{a}-\mathbf{r}$ with thionyl chloride in chloroform led to the corresponding chlorides $3 \mathbf{a}-\mathbf{r}$ with 
quantitative yields. Then, via chlorination of aromatic methyl group with trichloroisocyanuric acid (TCICA) in chloroform dichloro derivatives $4 \mathbf{a}-\mathbf{r}$ were obtained. The use of other chlorinating agents (N-chlorosuccinimide, sulfuryl chloride), varying solvents (methylene chloride, tetrachloroethane), and radical reactions initiators (AIBN, ACHN, benzoyl peroxide) did not lead to desired products. At the last stage, in reaction of dichlorides $4 \mathbf{a}-\mathbf{r}$ with a two-fold molar excess of tertiary amines ( $N, N$-dimethyoctylamine, $N, N$-dimethyldecylamine, $N, N$-dimethyldodecylamine, $N, N$-dimethyltetradecylamine, $N, N$-dimethylhexadecylamine, $N, N$-dimethyloctadecylamine) in ethanol at $70{ }^{\circ} \mathrm{C} 108$ bis-ammonium compounds $\mathbf{5} \mathbf{a}_{\mathbf{8}}-\mathbf{5} \mathbf{r}_{\mathbf{1 8}}$ were obtained. The substituent's variations allowed varying compound's lipophilicity and, thus, investigate the lipophilicity-activity relationships.

\subsection{Antibacterial Activity}

The antibacterial activity of 108 newly synthesized compounds was evaluated against three Gram-positive and three Gram-negative bacterial strains. Table 1 shows MICs of compounds in comparison with miramistin, benzalkonium chloride, and chlorhexidine. The lipophilicity of the synthesized ammonium salts was expressed in terms of their partition coefficient values $(\log \mathrm{P})$ calculated using online platform Chemicalize (ChemAxon) [20].

All compounds with tetradecyl, hexadecyl, and octadecyl substituents at the quaternary nitrogens were almost inactive regardless of the substituent on the acetal carbon atom. For compounds with small substituents at acetal carbon $\left(\mathrm{H}, \mathrm{CH}_{3}, \mathrm{C}_{2} \mathrm{H}_{5}, \mathrm{C}_{3} \mathrm{H}_{7}, \mathrm{CH}\left(\mathrm{CH}_{3}\right)_{2}\right)$ against all bacterial strains, the most active were compounds with decyl substituents at quaternary nitrogens $\left(\mathbf{5} \mathbf{a}_{\mathbf{1 0}}, \mathbf{5} \mathbf{b}_{\mathbf{1 0}}, \mathbf{5} \mathbf{c}_{\mathbf{1 0}}, \mathbf{5} \mathbf{d}_{\mathbf{1 0}}, \mathbf{5} \mathbf{e}_{\mathbf{1 0}}\right.$, MICs $0.5-8 \mu \mathrm{g} / \mathrm{mL}$ on Gram-positive and 2-64 $\mu \mathrm{g} / \mathrm{mL}$ on Gram-negative strains). Compounds with dodecyl substituents were slightly less active $\left(\mathbf{5 a}_{\mathbf{1 2}}, \mathbf{5} \mathbf{b}_{\mathbf{1 2}}, \mathbf{5} \mathbf{c}_{\mathbf{1 2}}, \mathbf{5} \mathbf{d}_{\mathbf{1 2}}\right.$, and $\left.\mathbf{5} \mathbf{e}_{\mathbf{1 2}}\right)$ and compounds with octyl substituents $\left(\mathbf{5} \mathbf{a}_{8}, \mathbf{5} \mathbf{b}_{8}, \mathbf{5} \mathbf{c}_{8}, \mathbf{5} \mathbf{d}_{8}\right.$, and $\left.\mathbf{5} \mathbf{e}_{8}\right)$ were inactive.

Due to an increase of the length of substituents on acetal carbon $\left(\mathrm{C}_{4} \mathrm{H}_{9}, \mathrm{C}\left(\mathrm{CH}_{3}\right)_{3}, \mathrm{CH}\left(\mathrm{CH}_{3}\right) \mathrm{C}_{2} \mathrm{H}_{5}\right.$, $\mathrm{C}_{5} \mathrm{H}_{11}$, and $\left.\mathrm{CH}\left(\mathrm{C}_{2} \mathrm{H}_{5}\right) \mathrm{C}_{2} \mathrm{H}_{5}, \mathrm{C}_{6} \mathrm{H}_{13}\right)$, the most active were compounds with decyl substituents at quaternary nitrogens $\left(\mathbf{5} \mathbf{f}_{\mathbf{1 0}}, \mathbf{5} \mathbf{g}_{\mathbf{1 0}}, \mathbf{5 h}_{\mathbf{1 0}}, \mathbf{5} \mathbf{i}_{\mathbf{1 0}}, \mathbf{5} \mathbf{j}_{\mathbf{1 0}}, \mathbf{5} \mathbf{k}_{\mathbf{1 0}}, \mathbf{5} \mathbf{l}_{\mathbf{1 0}}, \mathrm{MICs} \mathbf{0 . 2 5}-4 \mu \mathrm{g} / \mathrm{mL}\right.$ on Gram-positive and 0.5-8 $\mu \mathrm{g} / \mathrm{mL}$ on Gram-negative strains). Compounds with octyl and dodecyl substituents had similar activity and were less active than compounds with decyl substituents. A similar activity pattern was observed for compounds with a cyclohexyl substitutent at acetal carbon $\left(5 \mathbf{r}_{8}-5 \mathbf{r}_{12}\right)$.

With the further increase in the length of the substituents at the acetal carbon $\left(\mathrm{C}_{7} \mathrm{H}_{15}\right.$, $\left.\mathrm{CH}\left(\mathrm{C}_{2} \mathrm{H}_{5}\right) \mathrm{C}_{4} \mathrm{H}_{9}, \mathrm{C}_{8} \mathrm{H}_{17}, \mathrm{CH}\left(\mathrm{CH}_{3}\right) \mathrm{C}_{9} \mathrm{H}_{19}\right)$, compounds with octyl substituents at quaternary nitrogens $\left(5 \mathrm{~m}_{8}, \mathbf{5} \mathbf{n}_{\mathbf{8}}, \mathbf{5} \mathbf{o}_{\mathbf{8}}, \mathbf{5} \mathbf{p}_{\mathbf{8}}, \mathbf{5} \mathbf{q}_{\mathbf{8}}\right)$ became the most active (MICs $0.5-2 \mu \mathrm{g} / \mathrm{mL}$ on Gram-positive and $1-16 \mu \mathrm{g} / \mathrm{mL}$ on Gram-negative strains). Compounds with decyl substituents were slightly less active $\left(\mathbf{5} \mathbf{m}_{10}, \mathbf{5} \mathbf{n}_{10}\right.$, $\mathbf{5 o}_{10}, \mathbf{5} \mathbf{p}_{10}$, and $\mathbf{5 q}_{10}$ ) and compounds with dodecyl substituents were almost inactive.

Based on the results of the primary screening, 13 compounds $\left(\mathbf{5 a}_{\mathbf{1 2}}, \mathbf{5} \mathbf{c}_{\mathbf{1 0}}, \mathbf{5} \mathbf{c}_{\mathbf{1 2}}, \mathbf{5} \mathbf{d}_{\mathbf{1 0}}, \mathbf{5} \mathbf{f}_{\mathbf{1 0}}, \mathbf{5} \mathbf{g}_{\mathbf{1 0}}\right.$, $5 \mathbf{i}_{10}, 5 \mathbf{l}_{10}, \mathbf{5} \mathrm{m}_{8}, \mathbf{5} \mathbf{n}_{10}, \mathbf{5 0 _ { 8 }}, 5 \mathrm{p}_{8}$, and $\mathbf{5} \mathrm{q}_{8}$ in bold in Table 1) with an activity similar or superior when compared reference drugs were selected for further in-depth investigation for antibacterial activity, antifungal activity, and cytotoxicity in vitro.

The results of investigation of antibacterial activity on clinical Gram-positive and Gram-negative

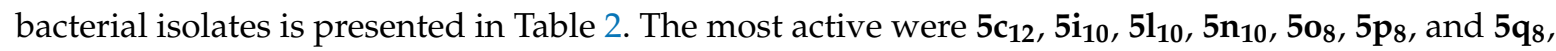
whose activity significantly exceeded that of all reference drugs. Compounds $\mathbf{5} \mathbf{a}_{\mathbf{1 2}}, \mathbf{5} \mathbf{f}_{\mathbf{1 0}}, \mathbf{5} \mathbf{g}_{\mathbf{1 0}}$, and $\mathbf{5 m}_{\mathbf{8}}$ exhibited, in general, antibacterial activity comparable to that of benzalkonium chloride and significantly exceeded miramistin and chlorhexidine in activity. Compounds $\mathbf{5} \mathbf{c}_{\mathbf{1 0}}$ and $\mathbf{5} \mathbf{d}_{\mathbf{1 0}}$ were almost inactive on all strains besides S. aureus 25 and S. intermedius 1143 MRSI (methicillin-resistant Staphylococcus intermedius). 
<smiles>[R7]C1([R7])OCc2c(COCC)cnc(C)c2O1</smiles>

2a-r $(55-86 \%)$

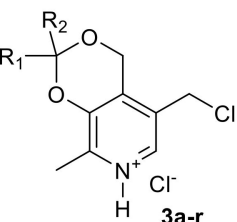

$(\sim 100 \%)$

iv, $v$

$\downarrow$

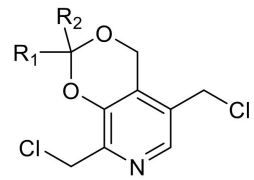

4a-r (39-68\%)

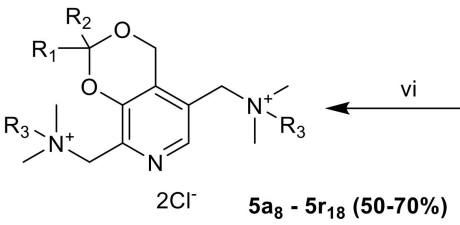

5a $a_{8} \cdot \mathrm{R}_{1}=\mathrm{R}_{2}=\mathrm{H}, \mathrm{R}_{3}=\mathrm{C}_{8} \mathrm{H}_{17}$ $5 a_{10} \cdot R_{1}=R_{2}=H, R_{3}=C_{10} H_{21}$ $5 a_{12} \cdot R_{1}=R_{2}=H, R_{3}=C_{12} \mathrm{H}_{25}$ $5 a_{14} \cdot R_{1}=R_{2}=H, R_{3}=C_{14} H_{29}$ $5 a_{16} \cdot R_{1}=R_{2}=H, R_{3}=C_{16} H_{33}$ 5a $a_{18} \cdot R_{1}=R_{2}=H, R_{3}=C_{18} H_{37}$

$\mathbf{5 b}_{\mathbf{8}} \cdot \mathrm{R}_{\mathbf{1}}=\mathrm{H}, \mathrm{R}_{2}=\mathrm{CH}_{3}, \mathrm{R}_{3}=\mathrm{C}_{8} \mathrm{H}_{17}$ $\mathbf{5 b}_{10} \cdot \mathrm{R}_{1}=\mathrm{H}, \mathrm{R}_{2}=\mathrm{CH}_{3}, \mathrm{R}_{3}=\mathrm{C}_{10} \mathrm{H}_{21}$ 5 $\mathbf{b}_{12} . \mathrm{R}_{1}=\mathrm{H}, \mathrm{R}_{2}=\mathrm{CH}_{3}, \mathrm{R}_{3}=\mathrm{C}_{12} \mathrm{H}_{25}$ $\mathbf{5 b}_{\mathbf{1 4}} \cdot \mathrm{R}_{\mathbf{1}}=\mathrm{H}, \mathrm{R}_{2}=\mathrm{CH}_{3}, \mathrm{R}_{3}=\mathrm{C}_{14} \mathrm{H}_{29}$ $\mathbf{5 b}_{\mathbf{1 6}} \cdot \mathrm{R}_{1}=\mathrm{H}, \mathrm{R}_{2}=\mathrm{CH}_{3}, \mathrm{R}_{3}=\mathrm{C}_{16} \mathrm{H}_{33}$ $\mathbf{5 b}_{18} \cdot \mathrm{R}_{1}=\mathrm{H}, \mathrm{R}_{2}=\mathrm{CH}_{3}, \mathrm{R}_{3}=\mathrm{C}_{18} \mathrm{H}_{37}$

5c $c_{8} \cdot \mathrm{R}_{1}=\mathrm{H}, \mathrm{R}_{2}=\mathrm{C}_{2} \mathrm{H}_{5}, \mathrm{R}_{3}=\mathrm{C}_{8} \mathrm{H}_{17}$ $\mathbf{5 c}_{\mathbf{1 0}} \cdot \mathrm{R}_{1}=\mathrm{H}, \mathrm{R}_{2}=\mathrm{C}_{2} \mathrm{H}_{5}, \mathrm{R}_{3}=\mathrm{C}_{10} \mathrm{H}_{21}$ 5c $c_{12} . \mathrm{R}_{1}=\mathrm{H}, \mathrm{R}_{2}=\mathrm{C}_{2} \mathrm{H}_{5}, \mathrm{R}_{3}=\mathrm{C}_{12} \mathrm{H}_{25}$ 5c $c_{14} \cdot \mathrm{R}_{1}=\mathrm{H}, \mathrm{R}_{2}=\mathrm{C}_{2} \mathrm{H}_{5}, \mathrm{R}_{3}=\mathrm{C}_{14} \mathrm{H}_{29}$ $\mathbf{5 c}_{16} . \mathrm{R}_{1}=\mathrm{H}, \mathrm{R}_{2}=\mathrm{C}_{2} \mathrm{H}_{5}, \mathrm{R}_{3}=\mathrm{C}_{16} \mathrm{H}_{33}$ 5c $c_{18} . R_{1}=H, R_{2}=C_{2} H_{5}, R_{3}=C_{18} H_{37}$

$\mathbf{5 d}_{\mathbf{8}} \cdot \mathrm{R}_{1}=\mathrm{H}, \mathrm{R}_{2}=\mathrm{C}_{3} \mathrm{H}_{7}, \mathrm{R}_{3}=\mathrm{C}_{8} \mathrm{H}_{17}$ 5d $d_{10} \cdot R_{1}=H, R_{2}=C_{3} H_{7}, R_{3}=C_{10} H_{21}$ $\mathbf{5} \mathbf{d}_{\mathbf{1 2}} \cdot \mathrm{R}_{\mathbf{1}}=\mathrm{H}, \mathrm{R}_{\mathbf{2}}=\mathrm{C}_{3} \mathrm{H}_{7}, \mathrm{R}_{3}=\mathrm{C}_{12} \mathrm{H}_{25}$ $\mathbf{5 d}_{\mathbf{1 4}} \cdot \mathrm{R}_{1}=\mathrm{H}, \mathrm{R}_{2}=\mathrm{C}_{3} \mathrm{H}_{7}, \mathrm{R}_{3}=\mathrm{C}_{14} \mathrm{H}_{29}$ 5d $d_{16} \cdot R_{1}=\mathrm{H}, \mathrm{R}_{2}=\mathrm{C}_{3} \mathrm{H}_{7}, \mathrm{R}_{3}=\mathrm{C}_{16} \mathrm{H}_{33}$ $\mathbf{5 d}_{\mathbf{1 8}} \cdot \mathrm{R}_{\mathbf{1}}=\mathrm{H}, \mathrm{R}_{2}=\mathrm{C}_{3} \mathrm{H}_{7}, \mathrm{R}_{3}=\mathrm{C}_{18} \mathrm{H}_{37}$

5e $\mathbf{e}_{8} \cdot \mathrm{R}_{1}=\mathrm{H}, \mathrm{R}_{2}=\mathrm{CH}\left(\mathrm{CH}_{3}\right)_{2}, \mathrm{R}_{3}=\mathrm{C}_{8} \mathrm{H}_{17}$ $\mathbf{5 e}_{\mathbf{1 0}} \cdot \mathrm{R}_{\mathbf{1}}=\mathrm{H}, \mathrm{R}_{\mathbf{2}}=\mathrm{CH}\left(\mathrm{CH}_{3}\right)_{2}, \mathrm{R}_{\mathbf{3}}=\mathrm{C}_{10} \mathrm{H}_{2}$ $\mathbf{5 e}_{12} \cdot \mathrm{R}_{1}=\mathrm{H}, \mathrm{R}_{2}=\mathrm{CH}\left(\mathrm{CH}_{3}\right)_{2}, \mathrm{R}_{3}=\mathrm{C}_{12} \mathrm{H}_{25}$ 5e $\mathbf{e}_{14} \cdot \mathrm{R}_{1}=\mathrm{H}, \mathrm{R}_{2}=\mathrm{CH}\left(\mathrm{CH}_{3}\right)_{2}, \mathrm{R}_{3}=\mathrm{C}_{14} \mathrm{H}_{2}$ $\mathbf{5 e}_{16} \cdot \mathrm{R}_{1}=\mathrm{H}, \mathrm{R}_{2}=\mathrm{CH}\left(\mathrm{CH}_{3}\right)_{2}, \mathrm{R}_{3}=\mathrm{C}_{16} \mathrm{H}_{33}$ 5e $\mathbf{e}_{18} . \mathrm{R}_{1}=\mathrm{H}, \mathrm{R}_{2}=\mathrm{CH}\left(\mathrm{CH}_{3}\right)_{2}, \mathrm{R}_{3}=\mathrm{C}_{18} \mathrm{H}_{37}$

5f $f_{8} \cdot \mathrm{R}_{1}=\mathrm{H}, \mathrm{R}_{2}=\mathrm{C}_{4} \mathrm{H}_{9}, \mathrm{R}_{3}=\mathrm{C}_{8} \mathrm{H}_{17}$ $\mathbf{5 f}_{10} \cdot \mathrm{R}_{1}=\mathrm{H}, \mathrm{R}_{2}=\mathrm{C}_{4} \mathrm{H}_{9}, \mathrm{R}_{3}=\mathrm{C}_{10} \mathrm{H}_{21}$ $\mathbf{5 f}_{12} \cdot \mathrm{R}_{1}=\mathrm{H}, \mathrm{R}_{2}=\mathrm{C}_{4} \mathrm{H}_{9}, \mathrm{R}_{3}=\mathrm{C}_{12} \mathrm{H}_{25}$ $\mathbf{5 f}_{14} \cdot \mathrm{R}_{1}=\mathrm{H}, \mathrm{R}_{2}=\mathrm{C}_{4} \mathrm{H}_{9}, \mathrm{R}_{3}=\mathrm{C}_{14} \mathrm{H}_{29}$ 5f $f_{16} . \mathrm{R}_{1}=\mathrm{H}, \mathrm{R}_{2}=\mathrm{C}_{4} \mathrm{H}_{9}, \mathrm{R}_{3}=\mathrm{C}_{16} \mathrm{H}_{33}$ $\mathbf{5 f}_{\mathbf{1 8}} \cdot \mathrm{R}_{1}=\mathrm{H}, \mathrm{R}_{2}=\mathrm{C}_{4} \mathrm{H}_{9}, \mathrm{R}_{3}=\mathrm{C}_{18} \mathrm{H}_{37}$ $\mathbf{5 g}_{8} \cdot \mathrm{R}_{1}=\mathrm{H}, \mathrm{R}_{2}=\mathrm{C}\left(\mathrm{CH}_{3}\right)_{3}, \mathrm{R}_{3}=\mathrm{C}_{8} \mathrm{H}_{17}$ $\mathbf{5} \mathbf{g}_{10} \cdot \mathrm{R}_{1}=\mathrm{H}, \mathrm{R}_{2}=\mathrm{C}\left(\mathrm{CH}_{3}\right)_{3}, \mathrm{R}_{3}=\mathrm{C}_{10} \mathrm{H}_{21}$ $\mathbf{5 g}_{\mathbf{1 2}} \cdot \mathrm{R}_{1}=\mathrm{H}, \mathrm{R}_{2}=\mathrm{C}\left(\mathrm{CH}_{3}\right)_{3}, \mathrm{R}_{3}=\mathrm{C}_{12} \mathrm{H}_{25}$ $5 g_{14} \cdot R_{1}=\mathrm{H}, \mathrm{R}_{2}=\mathrm{C}\left(\mathrm{CH}_{3}\right)_{3}, \mathrm{R}_{3}=\mathrm{C}_{14} \mathrm{H}_{29}$ $\mathbf{5 g}_{16} \cdot \mathrm{R}_{1}=\mathrm{H}, \mathrm{R}_{2}=\mathrm{C}\left(\mathrm{CH}_{3}\right)_{3}, \mathrm{R}_{3}=\mathrm{C}_{16} \mathrm{H}_{33}$ $\mathbf{5 g}_{18} \cdot \mathrm{R}_{1}=\mathrm{H}, \mathrm{R}_{2}=\mathrm{C}\left(\mathrm{CH}_{3}\right)_{3}, \mathrm{R}_{3}=\mathrm{C}_{18} \mathrm{H}_{37}$

5 $\mathbf{h}_{\mathbf{8}} \cdot \mathrm{R}_{1}=\mathrm{H}, \mathrm{R}_{2}=\mathrm{CH}\left(\mathrm{CH}_{3}\right) \mathrm{C}_{2} \mathrm{H}_{5}, \mathrm{R}_{3}=\mathrm{C}_{8} \mathrm{H}_{17}$ $5 h_{10} . R_{1}=H, R_{2}=C H\left(C_{3}\right) C_{2} H_{5}, R_{3}=C_{10} H_{21}$ $5 h_{12} . R_{1}=\mathrm{H}, \mathrm{R}_{2}=\mathrm{CH}\left(\mathrm{CH}_{3}\right) \mathrm{C}_{2} \mathrm{H}_{5}, \mathrm{R}_{3}=\mathrm{C}_{12} \mathrm{H}_{25}$ $5 h_{14} \cdot R_{1}=H, R_{2}=\mathrm{CH}\left(\mathrm{CH}_{3}\right) \mathrm{C}_{2} \mathrm{H}_{5}, \mathrm{R}_{3}=\mathrm{C}_{14} \mathrm{H}_{29}$ $\mathbf{5 h}_{16} \cdot \mathrm{R}_{1}=\mathrm{H}, \mathrm{R}_{2}=\mathrm{CH}\left(\mathrm{CH}_{3}\right) \mathrm{C}_{2} \mathrm{H}_{5}, \mathrm{R}_{3}=\mathrm{C}_{16} \mathrm{H}_{33}$ 5h $h_{18} \cdot \mathrm{R}_{1}=\mathrm{H}, \mathrm{R}_{2}=\mathrm{CH}\left(\mathrm{CH}_{3}\right) \mathrm{C}_{2} \mathrm{H}_{5}, \mathrm{R}_{3}=\mathrm{C}_{18} \mathrm{H}_{37}$

5i $i_{8} \cdot R_{1}=H, R_{2}=C_{5} H_{11}, R_{3}=C_{8} H_{17}$ $5 i_{10} \cdot R_{1}=H, R_{2}=C_{5} H_{11}, R_{3}=C_{10} H_{21}$ 5i $i_{12} \cdot R_{1}=H, R_{2}=C_{5} H_{11}, R_{3}=C_{12} H_{25}$ $5 i_{14} \cdot R_{1}=H, R_{2}=C_{5} H_{11}, R_{3}=C_{14} \mathrm{H}_{29}$ $\mathbf{5 i}_{16} \cdot \mathrm{R}_{1}=\mathrm{H}, \mathrm{R}_{2}=\mathrm{C}_{5} \mathrm{H}_{11}, \mathrm{R}_{3}=\mathrm{C}_{16} \mathrm{H}_{33}$ 5i $i_{18} \cdot R_{1}=H, R_{2}=C_{5} H_{11}, R_{3}=C_{18} H_{37}$

5j. $\mathrm{j}_{\mathbf{8}}=\mathrm{H}, \mathrm{R}_{2}=\mathrm{CH}\left(\mathrm{CH}_{3}\right) \mathrm{C}_{3} \mathrm{H}_{7}, \mathrm{R}_{3}=\mathrm{C}_{8} \mathrm{H}_{17}$ $\mathbf{5 j}_{10} \cdot \mathrm{R}_{1}=\mathrm{H}, \mathrm{R}_{2}=\mathrm{CH}\left(\mathrm{CH}_{3}\right) \mathrm{C}_{3} \mathrm{H}_{7}, \mathrm{R}_{3}=\mathrm{C}_{10} \mathrm{H}_{21}$ 5j $\mathbf{j}_{\mathbf{1 2}} \cdot \mathrm{R}_{1}=\mathrm{H}, \mathrm{R}_{2}=\mathrm{CH}\left(\mathrm{CH}_{3}\right) \mathrm{C}_{3} \mathrm{H}_{7}, \mathrm{R}_{3}=\mathrm{C}_{12} \mathrm{H}_{25}$ 5j $_{14} \cdot \mathrm{R}_{1}=\mathrm{H}, \mathrm{R}_{2}=\mathrm{CH}\left(\mathrm{CH}_{3}\right) \mathrm{C}_{3} \mathrm{H}_{7}, \mathrm{R}_{3}=\mathrm{C}_{14} \mathrm{H}_{29}$ 5j $j_{16} . \mathrm{R}_{1}=\mathrm{H}, \mathrm{R}_{2}=\mathrm{CH}\left(\mathrm{CH}_{3}\right) \mathrm{C}_{3} \mathrm{H}_{7}, \mathrm{R}_{3}=\mathrm{C}_{16} \mathrm{H}_{33}$ 5j $j_{18} . \mathrm{R}_{1}=\mathrm{H}, \mathrm{R}_{2}=\mathrm{CH}\left(\mathrm{CH}_{3}\right) \mathrm{C}_{3} \mathrm{H}_{7}, \mathrm{R}_{3}=\mathrm{C}_{18} \mathrm{H}_{37}$

5 $\mathbf{k}_{8} \cdot \mathrm{R}_{1}=\mathrm{H}, \mathrm{R}_{2}=\mathrm{CH}\left(\mathrm{C}_{2} \mathrm{H}_{5}\right) \mathrm{C}_{2} \mathrm{H}_{5}, \mathrm{R}_{3}=\mathrm{C}_{8} \mathrm{H}_{17}$ $\mathbf{5 k}_{\mathbf{1 0}} \cdot \mathrm{R}_{1}=\mathrm{H}, \mathrm{R}_{2}=\mathrm{CH}\left(\mathrm{C}_{2} \mathrm{H}_{5}\right) \mathrm{C}_{2} \mathrm{H}_{5}, \mathrm{R}_{3}=\mathrm{C}_{10} \mathrm{H}_{21}$ $\mathbf{5 k}_{12} \cdot \mathrm{R}_{1}=\mathrm{H}, \mathrm{R}_{2}=\mathrm{CH}\left(\mathrm{C}_{2} \mathrm{H}_{5}\right) \mathrm{C}_{2} \mathrm{H}_{5}, \mathrm{R}_{3}=\mathrm{C}_{12} \mathrm{H}_{25}$ 5 $\mathbf{k}_{14} \cdot \mathrm{R}_{1}=\mathrm{H}, \mathrm{R}_{2}=\mathrm{CH}\left(\mathrm{C}_{2} \mathrm{H}_{5}\right) \mathrm{C}_{2} \mathrm{H}_{5}, \mathrm{R}_{3}=\mathrm{C}_{14} \mathrm{H}_{29}$ $\mathbf{5 k}_{16} \cdot \mathrm{R}_{1}=\mathrm{H}, \mathrm{R}_{2}=\mathrm{CH}\left(\mathrm{C}_{2} \mathrm{H}_{5}\right) \mathrm{C}_{2} \mathrm{H}_{5}, \mathrm{R}_{3}=\mathrm{C}_{16} \mathrm{H}_{33}$ 5k $\mathbf{k}_{18} . \mathrm{R}_{1}=\mathrm{H}, \mathrm{R}_{2}=\mathrm{CH}\left(\mathrm{C}_{2} \mathrm{H}_{5}\right) \mathrm{C}_{2} \mathrm{H}_{5}, \mathrm{R}_{3}=\mathrm{C}_{18} \mathrm{H}_{37}$

5I $8 . \mathrm{R}_{1}=\mathrm{H}, \mathrm{R}_{2}=\mathrm{C}_{6} \mathrm{H}_{13}, \mathrm{R}_{3}=\mathrm{C}_{8} \mathrm{H}_{17}$ $5 \mathbf{I}_{10} \cdot \mathrm{R}_{1}=\mathrm{H}, \mathrm{R}_{2}=\mathrm{C}_{6} \mathrm{H}_{13}, \mathrm{R}_{3}=\mathrm{C}_{10} \mathrm{H}_{21}$ 5I $\mathrm{I}_{12} \cdot \mathrm{R}_{1}=\mathrm{H}, \mathrm{R}_{2}=\mathrm{C}_{6} \mathrm{H}_{13}, \mathrm{R}_{3}=\mathrm{C}_{12} \mathrm{H}_{25}$ 5I $14 \cdot \mathrm{R}_{1}=\mathrm{H}, \mathrm{R}_{2}=\mathrm{C}_{6} \mathrm{H}_{13}, \mathrm{R}_{3}=\mathrm{C}_{14} \mathrm{H}_{29}$ 5I $\mathrm{I}_{16} \cdot \mathrm{R}_{1}=\mathrm{H}, \mathrm{R}_{2}=\mathrm{C}_{6} \mathrm{H}_{13}, \mathrm{R}_{3}=\mathrm{C}_{16} \mathrm{H}_{33}$ 5I $\mathrm{I}_{18} \cdot \mathrm{R}_{1}=\mathrm{H}, \mathrm{R}_{2}=\mathrm{C}_{6} \mathrm{H}_{13}, \mathrm{R}_{3}=\mathrm{C}_{18} \mathrm{H}_{37}$
$5 m_{8} \cdot \mathrm{R}_{1}=\mathrm{H}, \mathrm{R}_{2}=\mathrm{C}_{7} \mathrm{H}_{15}, \mathrm{R}_{3}=\mathrm{C}_{8} \mathrm{H}_{17}$ $5 m_{10} \cdot R_{1}=H, R_{2}=\mathrm{C}_{7} \mathrm{H}_{15}, \mathrm{R}_{3}=\mathrm{C}_{10} \mathrm{H}_{21}$ $5 m_{12} \cdot R_{1}=H, R_{2}=C_{7} H_{15}, R_{3}=C_{12} H_{25}$ $\mathbf{5 m}_{14} \cdot \mathrm{R}_{1}=\mathrm{H}, \mathrm{R}_{2}=\mathrm{C}_{7} \mathrm{H}_{15}, \mathrm{R}_{3}=\mathrm{C}_{14} \mathrm{H}_{29}$ $5 m_{16} \cdot \mathrm{R}_{1}=\mathrm{H}, \mathrm{R}_{2}=\mathrm{C}_{7} \mathrm{H}_{15}, \mathrm{R}_{3}=\mathrm{C}_{16} \mathrm{H}_{33}$ $\mathbf{5 m}_{18} \cdot \mathrm{R}_{1}=\mathrm{H}, \mathrm{R}_{2}=\mathrm{C}_{7} \mathrm{H}_{15}, \mathrm{R}_{3}=\mathrm{C}_{18} \mathrm{H}_{37}$

5n $\mathbf{n}_{8} . \mathrm{R}_{1}=\mathrm{H}, \mathrm{R}_{2}=\mathrm{CH}\left(\mathrm{C}_{2} \mathrm{H}_{5}\right) \mathrm{C}_{4} \mathrm{H}_{9}, \mathrm{R}_{3}=\mathrm{C}_{8} \mathrm{H}_{17}$ $\mathbf{5 n}_{10} \cdot \mathrm{R}_{1}=\mathrm{H}, \mathrm{R}_{2}=\mathrm{CH}\left(\mathrm{C}_{2} \mathrm{H}_{5}\right) \mathrm{C}_{4} \mathrm{H}_{9}, \mathrm{R}_{3}=\mathrm{C}_{10} \mathrm{H}_{21}$ $5 n_{12} \cdot \mathrm{R}_{1}=\mathrm{H}, \mathrm{R}_{2}=\mathrm{CH}\left(\mathrm{C}_{2} \mathrm{H}_{5}\right) \mathrm{C}_{4} \mathrm{H}_{9}, \mathrm{R}_{3}=\mathrm{C}_{12} \mathrm{H}_{25}$ $\mathbf{5 n}_{14} \cdot \mathrm{R}_{1}=\mathrm{H}, \mathrm{R}_{2}=\mathrm{CH}\left(\mathrm{C}_{2} \mathrm{H}_{5}\right) \mathrm{C}_{4} \mathrm{H}_{9}, \mathrm{R}_{3}=\mathrm{C}_{14} \mathrm{H}_{29}$ $\mathbf{5 n}_{\mathbf{1 6}} \cdot \mathrm{R}_{\mathbf{1}}=\mathrm{H}, \mathrm{R}_{2}=\mathrm{CH}\left(\mathrm{C}_{2} \mathrm{H}_{5}\right) \mathrm{C}_{4} \mathrm{H}_{9}, \mathrm{R}_{3}=\mathrm{C}_{16} \mathrm{H}_{33}$ $5 n_{18} \cdot R_{1}=\mathrm{H}, \mathrm{R}_{2}=\mathrm{CH}\left(\mathrm{C}_{2} \mathrm{H}_{5}\right) \mathrm{C}_{4} \mathrm{H}_{9}, \mathrm{R}_{3}=\mathrm{C}_{18} \mathrm{H}_{37}$

5o $\mathbf{o}_{8} \cdot \mathrm{R}_{1}=\mathrm{H}, \mathrm{R}_{2}=\mathrm{C}_{8} \mathrm{H}_{17}, \mathrm{R}_{3}=\mathrm{C}_{8} \mathrm{H}_{17}$ $5_{0_{10}} \cdot \mathrm{R}_{1}=\mathrm{H}, \mathrm{R}_{2}=\mathrm{C}_{8} \mathrm{H}_{17}, \mathrm{R}_{3}=\mathrm{C}_{10} \mathrm{H}_{21}$ $5_{0_{12}} \cdot \mathrm{R}_{1}=\mathrm{H}, \mathrm{R}_{2}=\mathrm{C}_{8} \mathrm{H}_{17}, \mathrm{R}_{3}=\mathrm{C}_{12} \mathrm{H}_{25}$ 5o $14 . \mathrm{R}_{1}=\mathrm{H}, \mathrm{R}_{2}=\mathrm{C}_{8} \mathrm{H}_{17}, \mathrm{R}_{3}=\mathrm{C}_{14} \mathrm{H}_{29}$ $5_{\mathbf{o}_{16}} \cdot \mathrm{R}_{1}=\mathrm{H}, \mathrm{R}_{2}=\mathrm{C}_{8} \mathrm{H}_{17}, \mathrm{R}_{3}=\mathrm{C}_{16} \mathrm{H}_{33}$ $5_{0_{18}} \cdot \mathrm{R}_{1}=\mathrm{H}, \mathrm{R}_{2}=\mathrm{C}_{8} \mathrm{H}_{17}, \mathrm{R}_{3}=\mathrm{C}_{18} \mathrm{H}_{37}$

5p $\mathbf{p}_{8} \cdot \mathrm{R}_{1}=\mathrm{H}, \mathrm{R}_{2}=\mathrm{CH}\left(\mathrm{CH}_{3}\right) \mathrm{C}_{9} \mathrm{H}_{19}, \mathrm{R}_{3}=\mathrm{C}_{8} \mathrm{H}_{17}$ 5 $p_{10} . \mathrm{R}_{1}=\mathrm{H}, \mathrm{R}_{2}=\mathrm{CH}\left(\mathrm{CH}_{3}\right) \mathrm{C}_{9} \mathrm{H}_{19}, \mathrm{R}_{3}=\mathrm{C}_{10} \mathrm{H}_{21}$ 5p $p_{12} \cdot \mathrm{R}_{1}=\mathrm{H}, \mathrm{R}_{2}=\mathrm{CH}\left(\mathrm{CH}_{3}\right) \mathrm{C}_{9} \mathrm{H}_{19}, \mathrm{R}_{3}=\mathrm{C}_{12} \mathrm{H}_{25}$ $\mathbf{5 p}_{14} \cdot \mathrm{R}_{1}=\mathrm{H}, \mathrm{R}_{2}=\mathrm{CH}\left(\mathrm{CH}_{3}\right) \mathrm{C}_{9} \mathrm{H}_{19}, \mathrm{R}_{3}=\mathrm{C}_{14} \mathrm{H}_{29}$ 5p $p_{16} . \mathrm{R}_{1}=\mathrm{H}, \mathrm{R}_{2}=\mathrm{CH}\left(\mathrm{CH}_{3}\right) \mathrm{C}_{9} \mathrm{H}_{19}, \mathrm{R}_{3}=\mathrm{C}_{16} \mathrm{H}_{33}$ 5p $p_{18} . \mathrm{R}_{1}=\mathrm{H}, \mathrm{R}_{2}=\mathrm{CH}\left(\mathrm{CH}_{3}\right) \mathrm{C}_{9} \mathrm{H}_{19}, \mathrm{R}_{3}=\mathrm{C}_{18} \mathrm{H}_{37}$

5q $q_{8} \cdot \mathrm{R}_{1}=\mathrm{CH}_{3}, \mathrm{R}_{2}=\mathrm{C}_{8} \mathrm{H}_{17}, \mathrm{R}_{3}=\mathrm{C}_{8} \mathrm{H}_{17}$ $\mathbf{5} \mathbf{q}_{10} \cdot \mathrm{R}_{1}=\mathrm{CH}_{3}, \mathrm{R}_{2}=\mathrm{C}_{8} \mathrm{H}_{17}, \mathrm{R}_{3}=\mathrm{C}_{10} \mathrm{H}_{2}$ $\mathbf{5 q}_{12} \cdot \mathrm{R}_{1}=\mathrm{CH}_{3}, \mathrm{R}_{2}=\mathrm{C}_{8} \mathrm{H}_{17}, \mathrm{R}_{3}=\mathrm{C}_{12} \mathrm{H}_{25}$ $5 q_{14} \cdot R_{1}=\mathrm{CH}_{3}, \mathrm{R}_{2}=\mathrm{C}_{8} \mathrm{H}_{17}, \mathrm{R}_{3}=\mathrm{C}_{14} \mathrm{H}_{29}$ $\mathbf{5} \mathbf{q}_{16} \cdot \mathrm{R}_{1}=\mathrm{CH}_{3}, \mathrm{R}_{2}=\mathrm{C}_{8} \mathrm{H}_{17}, \mathrm{R}_{3}=\mathrm{C}_{16} \mathrm{H}_{33}$ $\mathbf{5 q}_{18} \cdot \mathrm{R}_{1}=\mathrm{CH}_{3}, \mathrm{R}_{2}=\mathrm{C}_{8} \mathrm{H}_{17}, \mathrm{R}_{3}=\mathrm{C}_{18} \mathrm{H}_{37}$

5r $r_{8} . \mathrm{R}_{1}+\mathrm{R}_{2}=-\left(\mathrm{CH}_{2}\right)_{5^{-}}, \mathrm{R}_{3}=\mathrm{C}_{8} \mathrm{H}_{17}$ $5 r_{10} \cdot R_{1}+R_{2}=-\left(C_{2}\right)_{5^{-}}, R_{3}=\mathrm{C}_{10} \mathrm{H}_{21}$ 5r $\mathbf{r}_{12} \cdot \mathrm{R}_{1}+\mathrm{R}_{2}=-\left(\mathrm{CH}_{2}\right)_{5^{-}}, \mathrm{R}_{3}=\mathrm{C}_{12} \mathrm{H}_{25}$ $5 r_{14} \cdot R_{1}+R_{2}=-\left(C_{2}\right)_{5^{-}}, R_{3}=C_{14} H_{29}$ $5 r_{16} \cdot R_{1}+R_{2}=-\left(C_{2}\right)_{5^{-}}, R_{3}=C_{16} H_{33}$ $5 r_{18} \cdot R_{1}+R_{2}=-\left(C_{2}\right)_{5^{-}}, R_{3}=C_{18} H_{37}$

Scheme 1. Reagents and conditions. (i) $\mathrm{R}_{1} \mathrm{C}(\mathrm{O}) \mathrm{R}_{2}, \mathrm{HCl}, 3-5^{\circ} \mathrm{C}, 3 \mathrm{~h}$; or $\mathrm{C}_{6} \mathrm{H}_{6}, \mathrm{R}_{1} \mathrm{C}(\mathrm{O}) \mathrm{R}_{2}, p$ - $\mathrm{TsOH}$, reflux, $17 \mathrm{~h}$, (ii) $\mathrm{H}_{2} \mathrm{O}, \mathrm{NaOH}, 2{ }^{\circ} \mathrm{C}$, (iii) $\mathrm{CH}_{2} \mathrm{Cl}_{2}, \mathrm{SOCl}_{2}, 20{ }^{\circ} \mathrm{C}, 17 \mathrm{~h}$, (iv) $\mathrm{H}_{2} \mathrm{O} / \mathrm{CHCl}_{3}, \mathrm{NaHCO}_{3}$, (v) $\mathrm{CHCl}_{3}$, TCICA, reflux, $4 \mathrm{~h}$, (vi) $\mathrm{C}_{2} \mathrm{H}_{5} \mathrm{OH}, \mathrm{R}_{3} \mathrm{~N}\left(\mathrm{CH}_{3}\right)_{2}, 70{ }^{\circ} \mathrm{C}, 5 \mathrm{~h}$. 
Table 1. In vitro antibacterial activity of novel bis-ammonium salts of pyridoxine ${ }^{\text {a }}$.

\begin{tabular}{|c|c|c|c|c|c|c|c|}
\hline \multirow{3}{*}{ Compound } & \multicolumn{6}{|c|}{ MICs $(\mu \mathrm{g} / \mathrm{mL})$} & \multirow{3}{*}{$\log P$} \\
\hline & \multicolumn{3}{|c|}{ Gram-Positive Bacteria } & \multicolumn{3}{|c|}{ Gram-Negative Bacteria } & \\
\hline & S. aureus ATCC 29213 & S. epidermidis(clinical) & B. subtilis 168 & E. coli ATCC 25922 & К. pneumoniae 1813 & $\begin{array}{l}\text { P. aeruginosa } \\
\text { ATCC } 27853\end{array}$ & \\
\hline $5 a_{8}$ & 64 & 64 & 64 & $>64$ & 64 & $>64$ & -1.74 \\
\hline $5 a_{10}$ & 2 & 2 & 2 & 32 & 2 & $>64$ & 0.03 \\
\hline $5 a_{12}$ & 4 & 2 & 8 & 16 & 4 & 32 & 1.81 \\
\hline $5 a_{14}$ & 32 & 64 & $>64$ & $>64$ & 64 & $>64$ & 3.59 \\
\hline $5 a_{16}$ & $>64$ & $>64$ & $>64$ & $>64$ & $>64$ & $>64$ & 5.37 \\
\hline $5 a_{18}$ & $>64$ & $>64$ & $>64$ & $>64$ & $>64$ & $>64$ & 7.15 \\
\hline $5 b_{8}$ & 64 & $>64$ & $>64$ & $>64$ & $>64$ & $>64$ & -1.51 \\
\hline $5 b_{10}$ & 2 & 2 & 2 & 32 & 2 & 64 & 0.26 \\
\hline $5 b_{12}$ & 8 & 1 & 8 & 16 & 4 & 16 & 2.04 \\
\hline $5 b_{14}$ & $>64$ & $>64$ & $>64$ & $>64$ & $>64$ & $>64$ & 3.82 \\
\hline $5 b_{16}$ & 64 & 64 & 64 & 64 & 64 & 64 & 5.60 \\
\hline $5 b_{18}$ & $>64$ & $>64$ & $>64$ & $>64$ & $>64$ & $>64$ & 7.38 \\
\hline $5 c_{8}$ & 32 & 32 & 64 & $>64$ & 32 & $>64$ & -0.88 \\
\hline $5 c_{10}$ & 0.5 & 0.5 & 32 & 8 & 1 & 16 & 0.90 \\
\hline $5 c_{12}$ & 1 & 2 & 4 & 16 & 4 & 32 & 2.68 \\
\hline $5 c_{14}$ & 16 & 16 & 64 & 64 & 64 & 64 & 4.46 \\
\hline $5 c_{16}$ & $>64$ & $>64$ & $>64$ & $>64$ & $>64$ & $>64$ & 6.24 \\
\hline $5 c_{18}$ & $>64$ & $>64$ & $>64$ & $>64$ & $>64$ & $>64$ & 8.01 \\
\hline $5 d_{8}$ & 16 & 16 & $>64$ & $>64$ & 16 & $>64$ & -0.43 \\
\hline $5 d_{10}$ & 0.25 & 0.5 & 8 & 2 & 1 & 8 & 1.35 \\
\hline $5 d_{12}$ & 1 & $1-2$ & 2 & 16 & 2 & 16 & 3.12 \\
\hline $5 d_{14}$ & 16 & 16 & $>64$ & $>64$ & 64 & 64 & 4.90 \\
\hline $5 d_{16}$ & $>64$ & $>64$ & $>64$ & $>64$ & $>64$ & $>64$ & 6.68 \\
\hline $5 d_{16}$ & $>64$ & $>64$ & $>64$ & $>64$ & $>64$ & $>64$ & 8.46 \\
\hline $5 e_{8}$ & 32 & 32 & 32 & $>64$ & 32 & $>64$ & -0.40 \\
\hline Chlorhexidine & 2 & 1 & 1 & 1 & 2 & 4 & 4.51 \\
\hline Miramistin & 2 & 2 & 2 & 4 & $>64$ & 64 & 2.43 \\
\hline $\begin{array}{l}\text { Benzalkonium } \\
\text { chloride }\end{array}$ & 1 & 2 & 0.5 & 1 & $>64$ & 64 & - \\
\hline $5 e_{10}$ & 2 & 1 & 2 & 8 & 1 & 16 & 1.38 \\
\hline $5 e_{12}$ & 8 & 2 & 4 & 8 & 8 & 32 & 3.16 \\
\hline $5 e_{14}$ & 16 & 32 & 64 & $>64$ & 32 & $>64$ & 4.94 \\
\hline
\end{tabular}


Table 1. Cont

\begin{tabular}{|c|c|c|c|c|c|c|c|}
\hline \multirow{3}{*}{ Compound } & \multicolumn{6}{|c|}{ MICs $(\mu \mathrm{g} / \mathrm{mL})$} & \multirow{3}{*}{$\log P$} \\
\hline & \multicolumn{3}{|c|}{ Gram-Positive Bacteria } & \multicolumn{3}{|c|}{ Gram-Negative Bacteria } & \\
\hline & S. aureus ATCC 29213 & S. epidermidis(clinical) & B. subtilis 168 & E. coli ATCC 25922 & K. pneumoniae 1813 & $\begin{array}{l}\text { P. aeruginosa } \\
\text { ATCC } 27853\end{array}$ & \\
\hline $5 e_{16}$ & $>64$ & $>64$ & $>64$ & $>64$ & $>64$ & $>64$ & 6.72 \\
\hline $5 \mathbf{e}_{18}$ & $>64$ & $>64$ & $>64$ & $>64$ & $>64$ & $>64$ & 8.49 \\
\hline $5 f_{8}$ & 4 & 8 & 64 & 64 & 8 & 64 & 0.01 \\
\hline $5 f_{10}$ & 0.25 & 0.5 & 4 & 2 & 1 & 4 & 1.79 \\
\hline $5 f_{12}$ & 1 & 1 & 2 & 32 & 4 & 32 & 3.57 \\
\hline $5 f_{14}$ & 32 & 64 & $>64$ & $>64$ & 64 & $>64$ & 5.35 \\
\hline $5 f_{16}$ & $>64$ & $>64$ & $>64$ & $>64$ & $>64$ & $>64$ & 7.13 \\
\hline $5 f_{18}$ & $>64$ & $>64$ & $>64$ & $>64$ & $>64$ & $>64$ & 8.90 \\
\hline $5 g_{8}$ & 8 & 16 & 8 & 64 & 8 & 64 & 0.09 \\
\hline $5 g_{10}$ & 0.5 & 0.5 & 1 & 2 & 0.5 & 8 & 1.84 \\
\hline $5 g_{12}$ & 1 & 1 & 4 & 2 & 2 & 8 & 3.65 \\
\hline $5 g_{14}$ & 32 & 32 & 64 & 64 & 64 & $>64$ & 5.43 \\
\hline $5 g_{16}$ & $>64$ & $>64$ & $>64$ & $>64$ & $>64$ & $>64$ & 7.21 \\
\hline $5 g_{18}$ & $>64$ & $>64$ & $>64$ & $>64$ & $>64$ & $>64$ & 8.99 \\
\hline $5 \mathbf{h}_{8}$ & 8 & 16 & 8 & 64 & 16 & $>64$ & 0.05 \\
\hline $5 h_{10}$ & 2 & 1 & 1 & 4 & 2 & 8 & 1.83 \\
\hline $5 h_{12}$ & 4 & 4 & 4 & 8 & 8 & 32 & 3.60 \\
\hline $5 h_{14}$ & 16 & $>64$ & $>64$ & $>64$ & $>64$ & $>64$ & 5.38 \\
\hline $5 h_{16}$ & $>64$ & $>64$ & $>64$ & $>64$ & $>64$ & $>64$ & 7.16 \\
\hline $5 h_{18}$ & $>64$ & $>64$ & $>64$ & $>64$ & $>64$ & $>64$ & 8.94 \\
\hline $5 i_{8}$ & 2 & 2 & 2 & 32 & 2 & 32 & 0.46 \\
\hline $5 \mathbf{i}_{10}$ & 0.5 & 0.5 & 2 & 1 & 1 & 4 & 2.24 \\
\hline $5 i_{12}$ & 1 & 2 & 8 & 16 & 8 & 32 & 4.01 \\
\hline $5 \mathbf{i}_{14}$ & 64 & 64 & $>64$ & $>64$ & $>64$ & $>64$ & 5.79 \\
\hline $5 \mathbf{i}_{16}$ & $>64$ & $>64$ & $>64$ & $>64$ & $>64$ & $>64$ & 7.57 \\
\hline $5 \mathbf{i}_{18}$ & $>64$ & $>64$ & $>64$ & $>64$ & $>64$ & $>64$ & 9.35 \\
\hline $5 \mathbf{j}_{8}$ & 4 & 4 & 4 & 64 & 4 & 64 & 0.49 \\
\hline $5 j_{10}$ & 1 & 1 & 1 & 2 & 2 & 4 & 2.27 \\
\hline Chlorhexidine & 2 & 1 & 1 & 1 & 2 & 4 & 4.51 \\
\hline Miramistin & 2 & 2 & 2 & 4 & $>64$ & 64 & 2.43 \\
\hline $\begin{array}{l}\text { Benzalkonium } \\
\text { chloride }\end{array}$ & 1 & 2 & 0.5 & 1 & $>64$ & 64 & - \\
\hline
\end{tabular}


Table 1. Cont

\begin{tabular}{|c|c|c|c|c|c|c|c|}
\hline \multirow{3}{*}{ Compound } & \multicolumn{6}{|c|}{$\operatorname{MICs}(\mu \mathrm{g} / \mathrm{mL})$} & \multirow{3}{*}{$\log P$} \\
\hline & \multicolumn{3}{|c|}{ Gram-Positive Bacteria } & \multicolumn{3}{|c|}{ Gram-Negative Bacteria } & \\
\hline & S. aureus ATCC 29213 & S. epidermidis(clinical) & B. subtilis 168 & E. coli ATCC 25922 & K. pneumoniae 1813 & $\begin{array}{c}\text { P. aeruginosa } \\
\text { ATCC } 27853\end{array}$ & \\
\hline $5 \mathbf{j}_{12}$ & 8 & 4 & 16 & 8 & 8 & 32 & 4.05 \\
\hline $5 \mathbf{j}_{14}$ & 32 & 64 & $>64$ & $>64$ & $>64$ & $>64$ & 5.83 \\
\hline $5 j_{16}$ & $>64$ & $>64$ & $>64$ & $>64$ & $>64$ & $>64$ & 7.60 \\
\hline $5 \mathbf{j}_{18}$ & $>64$ & $>64$ & $>64$ & $>64$ & $>64$ & $>64$ & 9.38 \\
\hline $5 \mathbf{k}_{8}$ & 4 & 4 & 4 & 64 & 4 & 64 & 0.49 \\
\hline $5 \mathrm{k}_{10}$ & 1 & 1 & 2 & 2 & 2 & 4 & 2.27 \\
\hline $5 k_{12}$ & 8 & 4 & 16 & 8 & 32 & 32 & 4.05 \\
\hline $5 k_{14}$ & 32 & 64 & $>64$ & $>64$ & $>64$ & $>64$ & 5.83 \\
\hline $5 k_{16}$ & $>64$ & $>64$ & $>64$ & $>64$ & $>64$ & $>64$ & 7.60 \\
\hline $5 \mathbf{k}_{18}$ & $>64$ & $>64$ & $>64$ & $>64$ & $>64$ & $>64$ & 9.38 \\
\hline $51_{8}$ & 1 & 1 & 8 & 1 & 16 & 1 & 0.90 \\
\hline $5 l_{10}$ & 0.5 & 2 & 1 & 0.5 & 4 & 0.5 & 2.68 \\
\hline $5 l_{12}$ & 8 & 4 & 16 & 16 & 16 & $>64$ & 4.46 \\
\hline $5 l_{14}$ & 32 & 32 & $>64$ & $>64$ & $>64$ & $>64$ & 6.24 \\
\hline $5 l_{16}$ & $>64$ & $>64$ & $>64$ & $>64$ & $>64$ & $>64$ & 8.01 \\
\hline $5 l_{18}$ & $>64$ & $>64$ & $>64$ & $>64$ & $>64$ & $>64$ & 9.79 \\
\hline $5 \mathrm{~m}_{8}$ & 0.5 & 0.5 & 1 & 2 & 0.5 & 16 & 1.35 \\
\hline $5 m_{10}$ & 1 & 1 & 2 & 2 & 4 & 8 & 3.12 \\
\hline $5 m_{12}$ & 8 & 16 & 32 & $>64$ & 32 & $>64$ & 4.90 \\
\hline $5 m_{14}$ & $>64$ & $>64$ & $>64$ & $>64$ & $>64$ & $>64$ & 6.68 \\
\hline $5 m_{16}$ & $>64$ & $>64$ & $>64$ & $>64$ & $>64$ & $>64$ & 8.46 \\
\hline $5 m_{18}$ & $>64$ & $>64$ & $>64$ & $>64$ & $>64$ & $>64$ & 10.24 \\
\hline $5 n_{8}$ & 1 & 1 & 1 & 8 & 1 & 16 & 1.38 \\
\hline $5 n_{10}$ & 1 & 1 & 2 & 4 & 2 & 4 & 3.16 \\
\hline $5 n_{12}$ & 8 & 16 & 32 & 32 & 32 & 64 & 4.94 \\
\hline $5 n_{14}$ & $>64$ & $>64$ & $>64$ & $>64$ & $>64$ & $>64$ & 6.72 \\
\hline $5 n_{16}$ & $>64$ & $>64$ & $>64$ & $>64$ & $>64$ & $>64$ & 8.49 \\
\hline $5 n_{18}$ & $>64$ & $>64$ & $>64$ & $>64$ & $>64$ & $>64$ & 10.27 \\
\hline $50_{8}$ & 1 & 1 & 2 & 2 & 1 & 8 & 2.24 \\
\hline $5 o_{10}$ & 8 & 2 & 8 & 16 & 4 & 16 & 4.01 \\
\hline $5 o_{12}$ & 16 & 8 & 64 & 32 & 32 & 64 & 5.79 \\
\hline
\end{tabular}


Table 1. Cont

\begin{tabular}{|c|c|c|c|c|c|c|c|}
\hline \multirow{3}{*}{ Compound } & \multicolumn{6}{|c|}{ MICs $(\mu \mathrm{g} / \mathrm{mL})$} & \multirow{3}{*}{$\log P$} \\
\hline & \multicolumn{3}{|c|}{ Gram-Positive Bacteria } & \multicolumn{3}{|c|}{ Gram-Negative Bacteria } & \\
\hline & S. aureus ATCC 29213 & S. epidermidis(clinical) & B. subtilis 168 & E. coli ATCC 25922 & K. pneumoniae 1813 & $\begin{array}{l}\text { P. aeruginosa } \\
\text { ATCC } 27853\end{array}$ & \\
\hline Chlorhexidine & 2 & 1 & 1 & 1 & 2 & 4 & 4.51 \\
\hline Miramistin & 2 & 2 & 2 & 4 & $>64$ & 64 & 2.43 \\
\hline $\begin{array}{l}\text { Benzalkonium } \\
\text { chloride }\end{array}$ & 1 & 2 & 0.5 & 1 & $>64$ & 64 & - \\
\hline $50_{14}$ & $>64$ & $>64$ & $>64$ & $>64$ & $>64$ & $>64$ & 7.57 \\
\hline $50_{16}$ & $>64$ & $>64$ & $>64$ & $>64$ & $>64$ & $>64$ & 9.35 \\
\hline $50_{18}$ & $>64$ & $>64$ & $>64$ & $>64$ & $>64$ & $>64$ & 11.13 \\
\hline $5 p_{8}$ & 2 & 2 & 2 & 4 & 4 & 8 & 3.16 \\
\hline $5 p_{10}$ & 8 & 8 & 32 & 32 & 64 & $>64$ & 4.94 \\
\hline $5 p_{12}$ & $>64$ & $>64$ & $>64$ & $>64$ & $>64$ & $>64$ & 6.72 \\
\hline $5 p_{14}$ & $>64$ & $>64$ & $>64$ & $>64$ & $>64$ & $>64$ & 8.49 \\
\hline $5 p_{16}$ & $>64$ & $>64$ & $>64$ & $>64$ & $>64$ & $>64$ & 10.27 \\
\hline $5 p_{18}$ & $>64$ & $>64$ & $>64$ & $>64$ & $>64$ & $>64$ & 12.05 \\
\hline $5 q_{8}$ & 2 & 0.5 & 2 & 2 & 1 & 8 & 2.04 \\
\hline $5 q_{10}$ & 2 & 2 & 4 & 8 & 4 & 8 & 3.82 \\
\hline $5 q_{12}$ & 16 & 32 & $>64$ & $>64$ & $>64$ & $>64$ & 5.60 \\
\hline $5 q_{14}$ & $>64$ & $>64$ & $>64$ & $>64$ & $>64$ & $>64$ & 7.37 \\
\hline $5 q_{16}$ & $>64$ & $>64$ & $>64$ & $>64$ & $>64$ & $>64$ & 9.15 \\
\hline $5 q_{18}$ & $>64$ & $>64$ & $>64$ & $>64$ & $>64$ & $>64$ & 10.93 \\
\hline $5 r_{8}$ & 16 & 16 & 16 & 64 & 16 & $>64$ & -0.01 \\
\hline $5 r_{10}$ & 2 & 1 & 1 & 2 & 1 & 16 & 1.77 \\
\hline $5 r_{12}$ & 8 & 2 & 4 & 16 & 4 & 16 & 3.55 \\
\hline $5 r_{14}$ & 32 & 64 & $>64$ & 64 & $>64$ & $>64$ & 5.32 \\
\hline $5 r_{16}$ & $>64$ & $>64$ & $>64$ & $>64$ & $>64$ & $>64$ & 7.10 \\
\hline $5 r_{18}$ & $>64$ & $>64$ & $>64$ & $>64$ & $>64$ & $>64$ & 8.88 \\
\hline Chlorhexidine & 2 & 1 & 1 & 1 & 2 & 4 & 4.51 \\
\hline Miramistin & 2 & 2 & 2 & 4 & $>64$ & 64 & 2.43 \\
\hline $\begin{array}{l}\text { Benzalkonium } \\
\text { chloride }\end{array}$ & 1 & 2 & 0.5 & 1 & $>64$ & 64 & - \\
\hline
\end{tabular}

${ }^{a}$ MICs for the most active compounds are highlighted in bold. 
Table 2. In vitro antimicrobial activity of novel bis-ammonium salts of pyridoxine on clinical bacterial isolates.

\begin{tabular}{|c|c|c|c|c|c|c|c|c|c|c|c|c|c|c|c|c|}
\hline \multirow{2}{*}{ Strains } & \multicolumn{16}{|c|}{$\operatorname{MICs}(\mu \mathrm{g} / \mathrm{mL})$} \\
\hline & $5 \mathbf{a}_{12}$ & $5 c_{10}$ & $5 c_{12}$ & $5 d_{10}$ & $5 f_{10}$ & $5 g_{10}$ & $5 i_{10}$ & $5 l_{10}$ & $5 m_{8}$ & $5 n_{10}$ & $5 o_{8}$ & $5 p_{8}$ & $5 q_{8}$ & Chlorhexidine & Benzalkonium Chloride & Miramistin \\
\hline S. haemolyticus $837 \mathrm{MRSH}$ & 16 & 64 & 4 & 64 & 16 & 16 & 8 & 4 & 32 & 4 & 8 & 4 & 8 & 64 & 32 & 64 \\
\hline S. aureus 967 MRSA & 16 & 64 & 4 & 64 & 16 & 16 & 8 & 4 & 32 & 4 & 8 & 4 & 8 & 64 & 32 & 64 \\
\hline S. aureus 983 MRSA & 16 & 64 & 4 & 64 & 16 & 16 & 8 & 4 & 32 & 4 & 8 & 4 & 8 & 64 & 32 & 64 \\
\hline S. aureus 25 & 2 & 1 & 4 & 2 & 1 & 4 & 2 & 4 & 1 & 2 & 1 & 2 & 1 & 2 & 2 & 16 \\
\hline S. intermedius 1143 MRSI & 2 & 2 & 2 & 4 & 2 & 2 & 2 & 2 & 2 & 2 & 1 & 1 & 1 & 4 & 4 & 32 \\
\hline S. aureus 2020 MRSA & 32 & $>64$ & 8 & 64 & 16 & 16 & 16 & 8 & 64 & 4 & 8 & 4 & 8 & 64 & 32 & 64 \\
\hline Moraxella sp.764 & 16 & 64 & 4 & 64 & 8 & 8 & 8 & 4 & 32 & 2 & 4 & 2 & 4 & $>64$ & 32 & 64 \\
\hline Moraxella sp.765 & 8 & 64 & 4 & 64 & 8 & 8 & 8 & 4 & 32 & 2 & 4 & 2 & 4 & $>64$ & 32 & 64 \\
\hline Moraxella sp.829 & 16 & 64 & 4 & 64 & 8 & 8 & 8 & 4 & 32 & 4 & 8 & 2 & 4 & $>64$ & 32 & 64 \\
\hline Acinetobacter spp. 1 & 32 & 64 & 8 & 64 & 16 & 16 & 16 & 4 & 64 & 4 & 8 & 8 & 8 & 64 & 32 & 64 \\
\hline Acinetobacter spp. 3 & 32 & 64 & 8 & 64 & 16 & 16 & 16 & 8 & 32 & 4 & 8 & 4 & 8 & 64 & 32 & 64 \\
\hline Pseudomonas spp. 5 & 16 & $>64$ & 4 & 64 & 16 & 16 & 8 & 4 & 64 & 4 & 8 & 4 & 8 & $>64$ & 32 & 64 \\
\hline Pseudomonas spp. 6 & 32 & 64 & 4 & 64 & 16 & 16 & 16 & 4 & 32 & 4 & 8 & 2 & 8 & $>64$ & 32 & 64 \\
\hline Klebsiella spp. 11 & 32 & 64 & 8 & 64 & 16 & 16 & 8 & 4 & 32 & 4 & 8 & 4 & 8 & $>64$ & 32 & $>64$ \\
\hline Klebsiella spp. 12 & 32 & $>64$ & 8 & $>64$ & 16 & 16 & 16 & 4 & 64 & 4 & 8 & 4 & 8 & $>64$ & 32 & 64 \\
\hline Proteus spp. 17 & $>64$ & $>64$ & 64 & $>64$ & 64 & 32 & 64 & $>64$ & $>64$ & $>64$ & 64 & $>64$ & 64 & $>64$ & 64 & $>64$ \\
\hline
\end{tabular}




\subsection{Structure-Activity Relationship (SAR)}

To identify quantitative relationships "structure-antibacterial activity" in a series of synthesized QACs based on vitamin B6 derivatives $5 \mathbf{a}_{8}-5 \mathbf{r}_{18}$, the dependences of antibacterial activity on calculated in ChemAxon lipophilicity [22] were evaluated (Figure 2, for S. epidermidis and K. pneumoniae 1813). A strong correlation between the lipophilicity and antimicrobial activity of quaternary ammonium pyridoxine derivatives on S. epidermidis and K. pneumonia was observed. Thus, all the most active compounds had $\log \mathrm{P}$ values in the range of $1-3$, while compounds with $\log \mathrm{P}>6$ and $\log \mathrm{P}<0$ were almost inactive. Similar relationships were found for the other studied bacteria. Apparently, relationships reflect the important features of the active compounds essential for their effective interaction with the hydrophobic membrane core of bacterial cells.

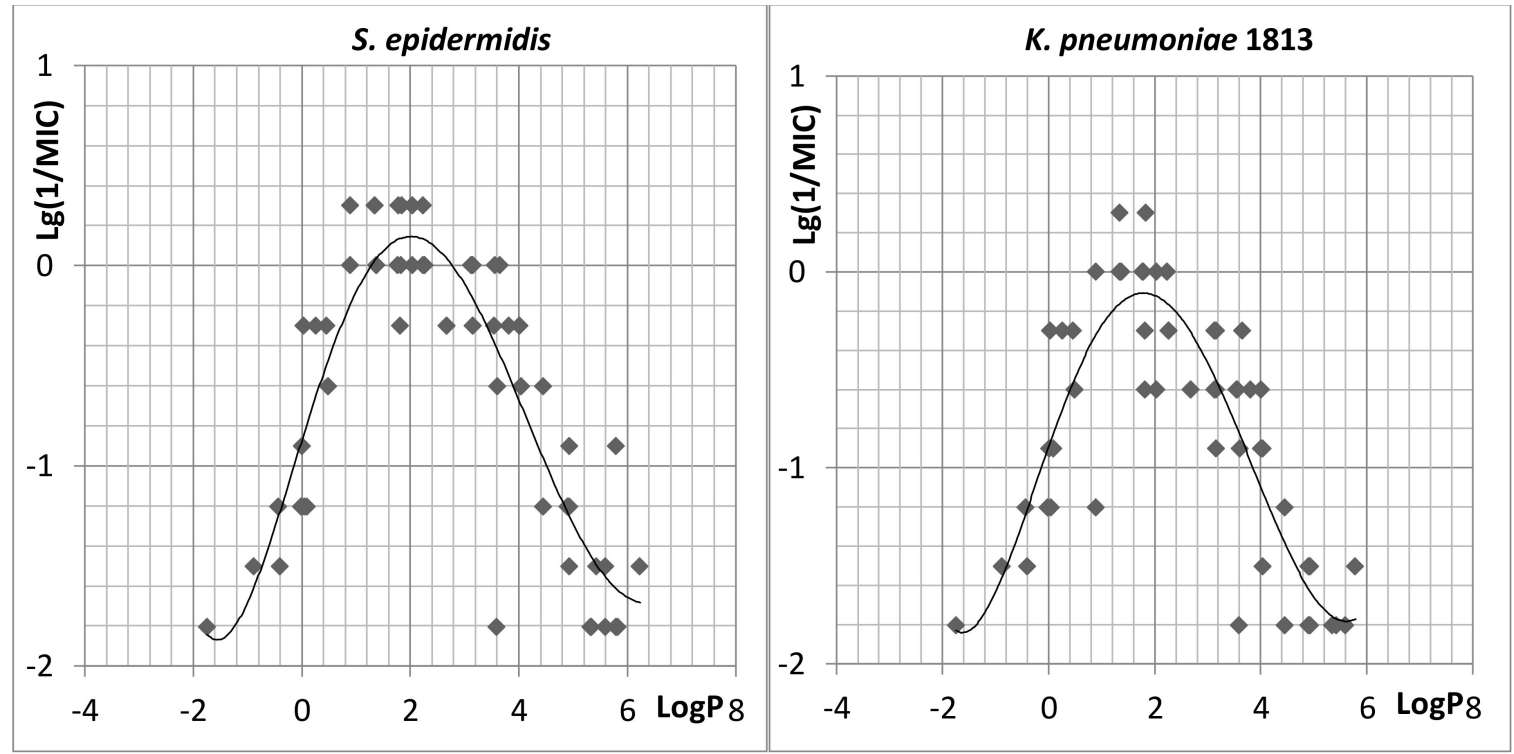

Figure 2. $\operatorname{Lg} 1 / \mathrm{MIC}$ versus calculated $\log \mathrm{P}$ relationship for novel bis-ammonium salts of pyridoxine. The parabolic line shows the polynomial approximation by using a second order model. Inactive compounds (MIC $>64 \mathrm{mg} / \mathrm{mL}$ ) are not shown.

\subsection{Antifungal Activity}

The antifungal activity is an important characteristic of antiseptics. Therefore, the effect of $\mathbf{5} \mathbf{a}_{12}$,

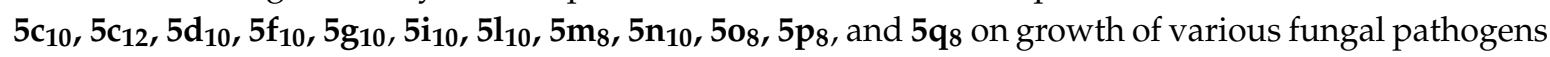
has been evaluated (Table 3 ).

Compound $\mathbf{5} \mathbf{c}_{10}, \mathbf{5} \mathbf{c}_{12}, \mathbf{5} \mathrm{d}_{10}, \mathbf{5} \mathrm{f}_{\mathbf{1 0}}, \mathbf{5} \mathbf{n}_{\mathbf{1 0}}$, and $\mathbf{5} \mathrm{p}_{\mathbf{8}}$ exhibited a pronounced antifungal activity against all tested fungi with minimum inhibitory concentrations (MIC) lower than all reference drugs. The highest activity demonstrated compound $5 \mathbf{d}_{10}$ with MIC values of $2-4 \mu \mathrm{g} / \mathrm{mL}$ against all tested fungi. Compounds $\mathbf{5} \mathbf{a}_{\mathbf{1 2}}, \mathbf{5} \mathbf{g}_{\mathbf{1 0}}, \mathbf{5} \mathbf{i}_{\mathbf{1 0}}, \mathbf{5} \mathbf{1}_{\mathbf{1 0}}, \mathbf{5 o}_{\mathbf{8}}$, and $\mathbf{5} \mathbf{q}_{\mathbf{8}}$ had a comparable activity with miramistin. Compound $5 \mathrm{~m}_{8}$ was almost inactive on all tested fungi similar to chlorhexidine.

\subsection{Cytotoxicity}

Cytotoxicity of compounds $5 \mathrm{a}_{12}, 5 \mathrm{c}_{10}, 5 \mathrm{c}_{12}, 5 \mathrm{~d}_{10}, 5 \mathrm{f}_{10}, 5 \mathrm{~g}_{10}, 5 \mathrm{i}_{10}, 5 \mathrm{l}_{10}, 5 \mathrm{~m}_{8}, 5 \mathrm{n}_{10}, 5 \mathrm{o}_{8}, 5 \mathrm{p}_{8}$, and $\mathbf{5} \mathrm{q}_{8}$ was evaluated on HEK-293 cells (Table 4). According to the results obtained, all compounds had values of cytotoxicity comparable with miramistin and chlorhexidine and were 2-4-fold less toxic when compared to benzalkonium chloride. 
Table 3. In vitro antifungal activity of novel bis-ammonium salts of pyridoxine.

\begin{tabular}{|c|c|c|c|c|}
\hline \multirow[b]{2}{*}{ Compound } & \multicolumn{4}{|c|}{ Minimum Inhibitory Concentrations (MICs) $(\mu \mathrm{g} / \mathrm{mL})$} \\
\hline & $\begin{array}{l}\text { Trichophyton } \\
\text { rubrum } 1336 \\
\text { (clinical) }\end{array}$ & $\begin{array}{c}\text { Aspergillus niger } \\
\text { F-1119 }\end{array}$ & $\begin{array}{l}\text { Candida albicans } \\
\text { NCTC- 885-653 }\end{array}$ & $\begin{array}{l}\text { Fusarium oxysporum } \\
\text { KM-19 (clinical) }\end{array}$ \\
\hline $5 \mathbf{a}_{12}$ & 32 & 16 & 16 & 32 \\
\hline $5 c_{10}$ & 16 & 8 & 4 & 8 \\
\hline $5 c_{12}$ & 16 & 8 & 4 & 8 \\
\hline $5 d_{10}$ & 2 & 4 & 2 & 4 \\
\hline $5 f_{10}$ & 16 & 16 & 8 & 16 \\
\hline $5 g_{10}$ & 32 & 32 & 32 & 32 \\
\hline $5 \mathbf{i}_{10}$ & 32 & 16 & 16 & 32 \\
\hline $5 l_{10}$ & 32 & 16 & 16 & 32 \\
\hline $5 m_{8}$ & 128 & 128 & 128 & 128 \\
\hline $5 n_{10}$ & 8 & 4 & 4 & 8 \\
\hline $50_{8}$ & 32 & 32 & 32 & 32 \\
\hline $5 p_{8}$ & 8 & 4 & 4 & 8 \\
\hline $5 q_{8}$ & 64 & 32 & 32 & 64 \\
\hline Miramistin & 32 & 32 & 32 & 32 \\
\hline Chlorhexidine & 128 & 128 & 64 & 128 \\
\hline Benzalkonium chloride & 16 & 8 & 8 & 16 \\
\hline
\end{tabular}

Table 4. Cytotoxicity of novel bis-ammonium salts of pyridoxine.

\begin{tabular}{cc}
\hline Compound & $\mathrm{CC}_{\mathbf{5 0}}, \boldsymbol{\mu g} / \mathbf{m L}$ (HEK-293) \\
\hline $\mathbf{5 a}_{\mathbf{1 2}}$ & $3.11 \pm 0.5$ \\
$\mathbf{5} \mathbf{c}_{\mathbf{1 0}}$ & $3.83 \pm 0.7$ \\
$\mathbf{5} \mathbf{c}_{\mathbf{1 2}}$ & $3.38 \pm 0.8$ \\
$\mathbf{5 d}_{\mathbf{1 0}}$ & $2.81 \pm 1.2$ \\
$\mathbf{5} \mathbf{f}_{\mathbf{1 0}}$ & $4.04 \pm 0.6$ \\
$\mathbf{5 g}_{\mathbf{1 0}}$ & $3.86 \pm 0.8$ \\
$\mathbf{5 i}_{\mathbf{1 0}}$ & $2.22 \pm 0.4$ \\
$\mathbf{5} \mathbf{l}_{\mathbf{1 0}}$ & $2.79 \pm 0.8$ \\
$\mathbf{5} \mathbf{m}_{\mathbf{8}}$ & $3.48 \pm 1.2$ \\
$\mathbf{5 n}_{\mathbf{1 0}}$ & $2.02 \pm 0.4$ \\
$\mathbf{5 \mathbf { o } _ { \mathbf { 8 } }}$ & $4.26 \pm 0.9$ \\
$\mathbf{5} \mathbf{p}_{\mathbf{8}}$ & $3.11 \pm 0.8$ \\
$\mathbf{5 q}_{\mathbf{8}}$ & $3.69 \pm 0.7$ \\
Miramistin & $6.83 \pm 1.1$ \\
Chlorhexidine & $5.48 \pm 1.5$ \\
Benzalkonium chloride & $1.02 \pm 0.4$ \\
\hline
\end{tabular}

\subsection{In Vivo Toxicity}

For the lead antibacterial and antifungal compounds $\mathbf{5} \mathbf{c}_{\mathbf{1 2}}, \mathbf{5} \mathbf{d}_{\mathbf{1 0}}$, and $\mathbf{5} \mathbf{p}_{\mathbf{8}}$ in vivo toxicity at oral administration on CD-1 mice were performed as described in Section 3.5 (Table 5). No remarkable findings were noted in both sexes in compound $\mathbf{5} \mathbf{c}_{\mathbf{1 2}}$ treatment group at necropsy. Compounds $\mathbf{5} \mathbf{d}_{\mathbf{1 0}}$ and $5 p_{8}$ treatment groups revealed stomach walls hypertrophy. Thus, all studied substances belong to category 4 of the globally harmonized system of classification and labeling of chemicals [23]. Compound $\mathbf{5} \mathbf{c}_{12}$ was considerably less toxic ( $\mathrm{LD}_{50} 1705 \mathrm{mg} / \mathrm{kg}$ ) than benzalkonium chloride ( $\mathrm{LD}_{50} 180 \mathrm{mg} / \mathrm{kg}$ [24]),

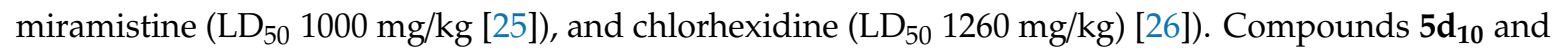
$5 p_{8}$ are 2 -fold and 4 -fold more toxic when compared to $5 c_{12}$. 
Table 5. Toxicology data obtained on oral administration of compounds $\mathbf{5} \mathbf{c}_{\mathbf{1 2}}, \mathbf{5} \mathbf{d}_{\mathbf{1 0}}$, and $\mathbf{5} \mathbf{p}_{\mathbf{8}}$ to CD-1 mice.

\begin{tabular}{cc}
\hline Compound & $\mathbf{L D}_{\mathbf{5 0}}, \mathbf{m g} / \mathbf{k g}$ \\
\hline $\mathbf{5 c}_{\mathbf{1 2}}$ & 1705 \\
$\mathbf{5 d}_{\mathbf{1 0}}$ & 809 \\
$\mathbf{5} \mathbf{p}_{\mathbf{8}}$ & 474 \\
Benzalkonium & $180[22]$ \\
chloride & $1000[23]$ \\
Miramistin & $1260[24]$ \\
Chlorhexidine & \\
\hline
\end{tabular}

Thus, the extensive screening of the antibacterial activity and toxicity of the modified structural analogs of compound $\mathbf{5} \mathbf{c}_{12}$ did not reveal compounds superior in antibacterial activity. In addition, its in vivo toxicity was significantly lower than that of compounds with comparable activity. For this reason, $\mathbf{5} \mathbf{c}_{12}$ was selected as a lead compound for further investigations.

\subsection{Anti-Biofilm Activity}

The anti-biofilm activity of $\mathbf{5} \mathbf{c}_{\mathbf{1 2}}$ has been tested on biofilms formed by S. aureus, S. epidermidis, E. coli, or P. aeruginosa. For that, 48-h-old bacterial biofilms prepared in 24-well plates were washed with sterile phosphate-buffered saline (PBS) and filled with $125 \mu \mathrm{L}$ of fresh basal medium (BM) broth containing $5 \mathbf{c}_{12}$, miramistin, or benzalkonium chloride in concentrations of their respective $8-16 \times \mathrm{MBC}$ (minimum bactericidal concentration, see Table 6 for values). After $24 \mathrm{~h}$ of incubation, the viability of biofilm-embedded cells was evaluated by colony-forming units (CFUs) counting.

Table 6. Bactericidal activity of compound $\mathbf{5} \mathbf{c}_{\mathbf{1 2}}$, benzalkonium chloride, and miramistin.

\begin{tabular}{cccc}
\hline Strain & \multicolumn{3}{c}{ MBC $(\boldsymbol{\mu g} / \mathbf{m L})$} \\
\cline { 2 - 4 } & $\mathbf{5 \mathbf { c } _ { \mathbf { 1 2 } }}$ & Benzalkonium Chloride & Miramistin \\
\hline S. aureus ATCC 29213 & 4 & 4 & 8 \\
S. epidermidis & 8 & 8 & 4 \\
P. aeruginosa ATCC 27853 & 128 & 128 & 128 \\
E. coli ATCC 25922 & 64 & 2 & 8 \\
\hline
\end{tabular}

In these experiments, $\mathbf{5} \mathbf{c}_{12}$ exhibited higher activity compared with the reference antimicrobials: a 3-log drop of CFUs of these bacteria was observed at $2 \times \mathrm{MBC}$ of $5 \mathrm{c}_{\mathbf{1 2}}$ (Figure 3), while $8 \times \mathrm{MBC}$ of both benzalkonium chloride and miramistin were required to obtain the same effect. Moreover, both reference antimicrobials were less active against $P$. aeruginosa biofilm even at $16 \times \mathrm{MBC}$, while $\mathbf{5} \mathbf{c}_{12}$ reduced the CFUs number by three orders of magnitude at $8 \times \mathrm{MBC}$ (Figure 3D). Thus, $\mathbf{5 c}_{\mathbf{1 2}}$ demonstrated anti-biofilm activity comparable or even higher than that of the benzalkonium chloride. 
A

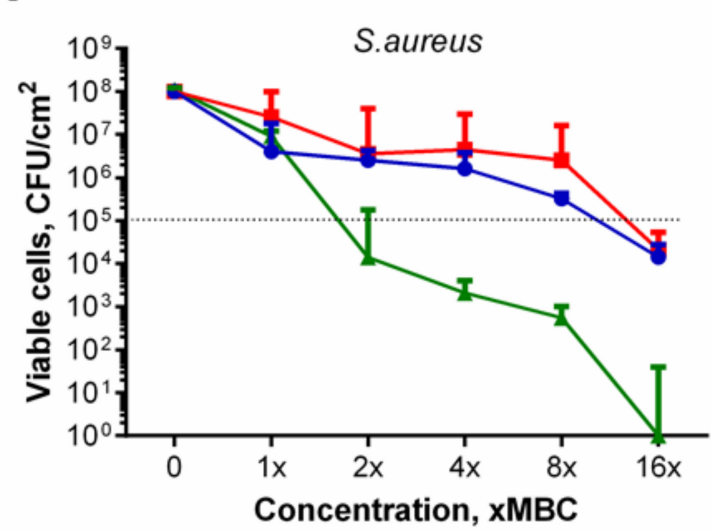

C

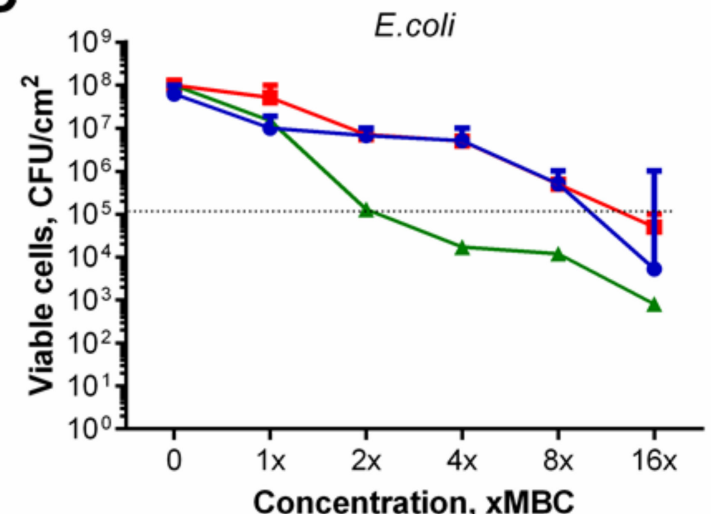

B

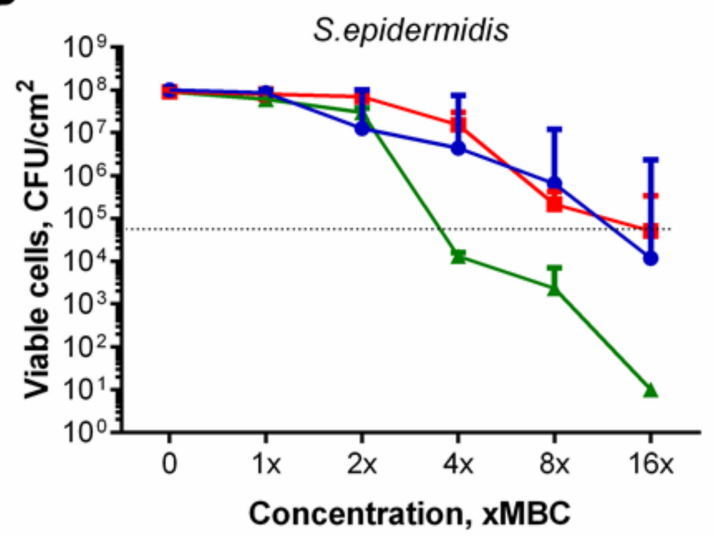

D

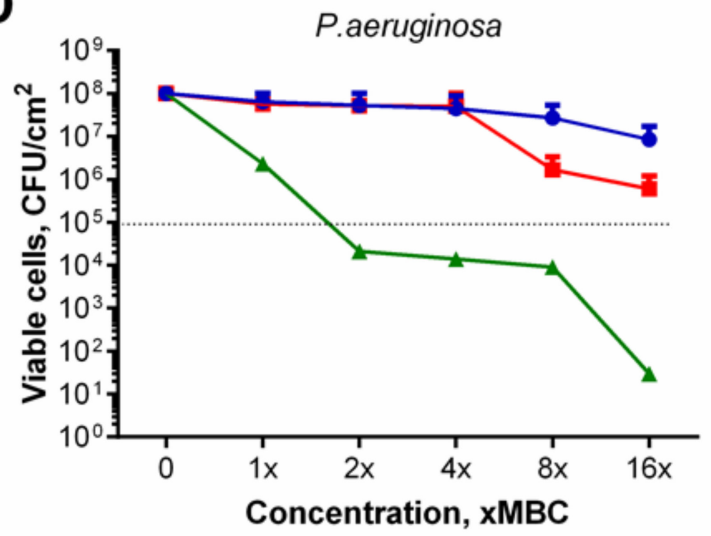

$\leftarrow 5 c_{12} \rightarrow$ Benzalkonium chloride $\rightarrow$ Miramistin

Figure 3. The effect of $\mathbf{5 c}_{\mathbf{1 2}}$ and reference antiseptics on biofilm-embedded bacteria. (A)-S. aureus; (B)-S. intermedius; (C) - E. coli, (D) - P. aeruginosa. The 48-h biofilms were treated with 1-16 $\times$ MBCs of benzalkonium chloride (circles), miramistin (squares), or $\mathbf{5} \mathbf{c}_{\mathbf{1 2}}$ (triangles) for $\mathbf{2 4} \mathrm{h}$ and then quantified by CFU counting. Data are present as medians from five independent experiments with interquartile range $(\mathrm{M} \pm \mathrm{IQR})$. Dotted line shows 3 log-decrease of viable cells.

\subsection{In Vivo Efficiency}

In vivo efficiency of $\mathbf{5} \mathbf{c}_{12}$ was estimated in a rat skin model in comparison with miramistin, benzalkonium chloride and chlorhexidine. Aqueous solution of $0.2 \% 5 \mathbf{c}_{12}$ reduced CFU (colony-forming unit) of E. coli CD CF-50 after 5 min of exposure on rat's skin (Table 7).

Table 7. In vivo antibacterial efficacy (Mean \pm SD) of $0.2 \%$ aqueous solution $\mathbf{5} \mathbf{c}_{\mathbf{1 2}}$, miramistin, benzalkonium chloride and chlorhexidine in a rat skin model, $n=6$.

\begin{tabular}{cc}
\hline Compound & Growth Inhibition Percentage $\left(\mathbf{C F U} / \mathbf{c m}^{\mathbf{2}}\right)$ \\
\hline $\mathbf{5 c}_{\mathbf{1 2}}$ & $97.5 \pm 1.7$ \\
Miramistin & $97.2 \pm 1.8$ \\
Benzalkonium chloride & $96.8 \pm 2.0$ \\
Chlorhexidine & $95.7 \pm 3.4$ \\
\hline
\end{tabular}

Compound $\mathbf{5} \mathbf{c}_{\mathbf{1 2}}$ demonstrated the strongest E. coli CD CF-50 growth inhibition (Figure 4). The growth inhibition percentage reached $97.5 \%$, which is comparable to reference drugs' efficiency [27]. 


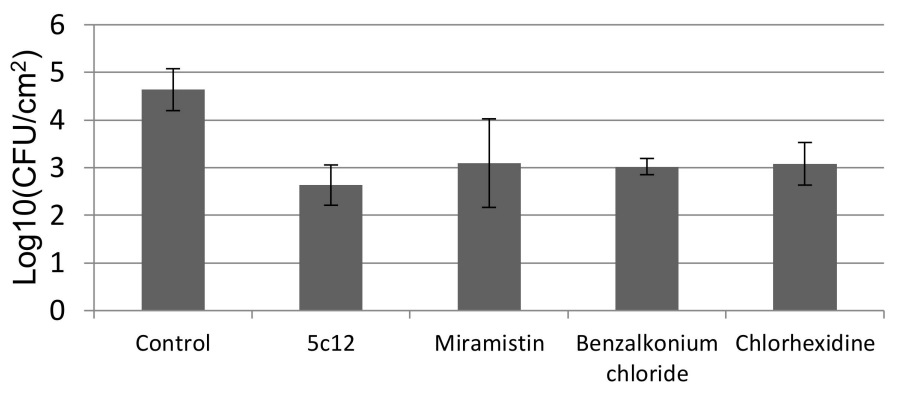

Figure 4. In vivo antibacterial efficacy test (Mean \pm SD) using a rat skin model. $\log 10$ number of viable cells in the control and the number of viable cells after exposure with $\mathbf{5} \mathbf{c}_{\mathbf{1 2}}$, miramistin, benzalkonium chloride, and chlorhexidine, $n=6$.

\subsection{Cytoplasmic Membrane Depolarization}

Quaternary ammonium salts are well known for their membrane-damaging action on bacterial cells [28]. To evaluate the effect of $\mathbf{5 c}_{\mathbf{1 2}}$ on the membrane polarization, the fluorescence of membrane-potential-sensitive cyanine dye $\mathrm{DiSC}_{3}(5)$ in suspensions of either S. aureus ATCC209p or E. coli CDCF-50 has been measured in the presence of $5 \mathbf{c}_{12}$ or either benzalkonium chloride or miramistin (Figure 5). In the presence of $\mathbf{5} \mathbf{c}_{12}$ and reference compounds, a rapid and intense increase of fluorescence of $\operatorname{DiSC}_{3}(5)$ has been observed in both S. aureus and E. coli suspensions. It was shown that the ability of $\mathbf{5 c}_{12}$ to change the electric potential of the bacterial membrane was comparable to the reference compounds. Significant differences between untreated cells and treated by $\mathbf{5 c}_{\mathbf{1 2}}(p \leq 0.05)$ cells were observed after 20 min of incubation (Figure $5 C, D)$. When the $\mathbf{5}_{\mathbf{1 2}}$ concentration increased from $1 \times \mathrm{MBC}$ to 2,4 , and $8 \times \mathrm{MBC}$, the membrane-damaging effect has been achieved two times faster. We found that a 10-min incubation of cells with $5 \mathbf{c}_{12}$ led to significant differences in the control (Figure 5A,B).
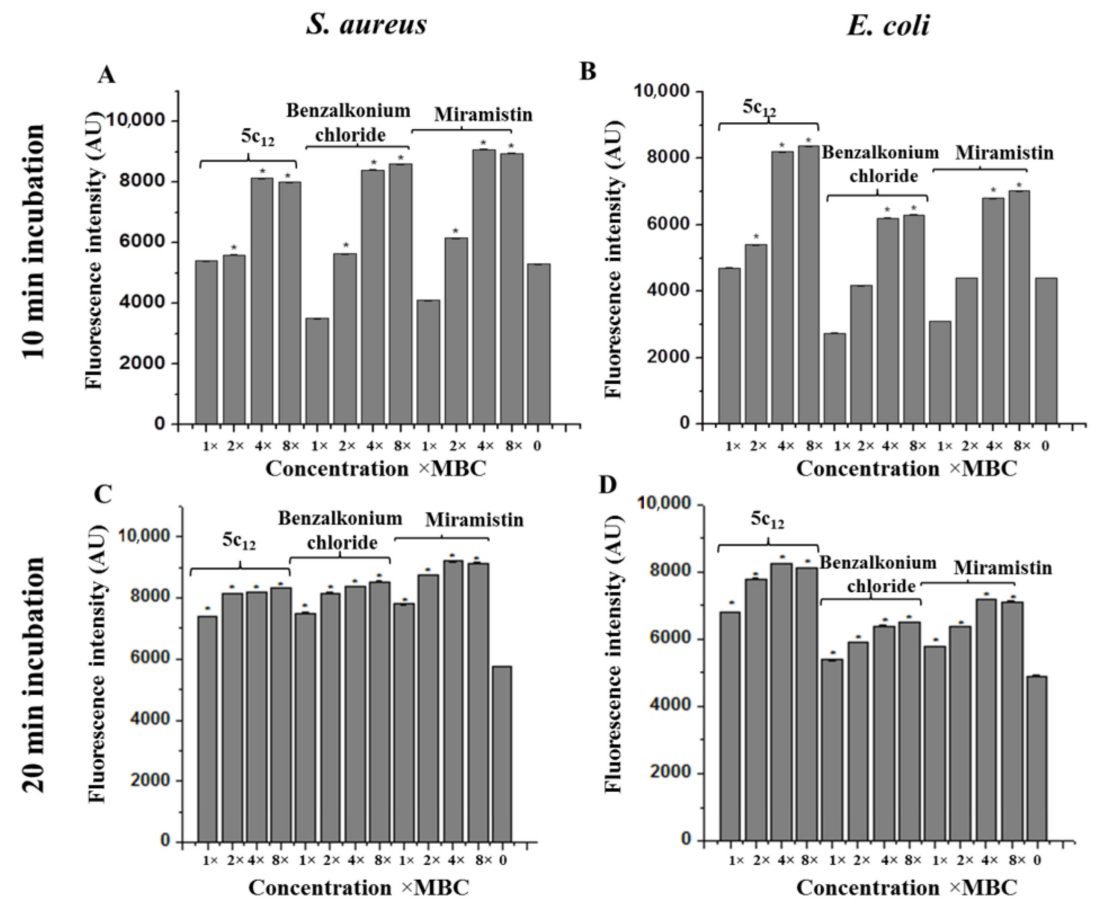

Figure 5. The effect of $\mathbf{5 c}_{12}$ on the electric potential of the bacterial membrane determined using a membrane-potential-sensitive cyanine dye $\operatorname{DiSC}_{3}(5)(10 \mathrm{~min}$ treatment by $1-8 \times \mathrm{MBC}(\mathbf{A}, \mathbf{B})$ and $20 \mathrm{~min}$ treatment by $1-8 \times \mathrm{MBC}(\mathbf{C}, \mathbf{D}))$. Asterisks denote a statistically significant difference in comparison with untreated cells. ${ }^{*}$ statistically significant difference from the control. 


\subsection{Outer Membrane Permeability}

Since the membrane depolarization can be driven with either membrane permeabilization or a metabolism drop, the ability of $\mathbf{5} \mathbf{c}_{\mathbf{1 2}}$ to permeate the bacterial outer membrane was determined using the fluorescent dye $N$-phenyl-1-naphthylamine (NPN). A strong dose-dependent response of $\mathbf{5 c}_{\mathbf{1 2}}$ on the Gram-negative bacteria outer membrane penetration was observed. For untreated E. coli cells, the permeability was $45 \%$. In the presence of $1 \times \mathrm{MBC}$ of $\mathbf{5 c}_{\mathbf{1 2}}$, the outer membrane permeability increased to $70 \%$ with increasing concentrations $(2 \times$ and $4 \times \mathrm{MBC})$ of $\mathbf{5 c}_{\mathbf{1 2}}$. The membrane permeability reached more than $80 \%$.

\subsection{Scanning Electron Microscopy}

The data presented above allow suggesting the destabilizing effect of $\mathbf{5 c}_{\mathbf{1 2}}$ on the bacterial membrane, which leads to an antiseptic effect of the drug. These assumptions are confirmed by the SEM images of treated cells (Figure 6).

Thus, while the untreated $S$. aureus cells had a spherical regular shape with a dense surface with small gentle tubercles without vortices, dead cells were not detected in the visual fields (Figure 6A). After $\mathbf{5}_{12}$ treatment, dead cells with a shrunken cell membrane were observed. The shape of most cells became non-spherical and irregular with a losing surface and organic matter on most of the cells, possibly due to damage of the cytoplasmic membrane and leakage of internal contents (Figure 6C). A number of cells exhibited deep creases, which indicated the folding of the cell wall into the cell due to the outflow of cytoplasm. In contrast to $S$. aureus, untreated $E$. coli cells had a wrinkled surface (Figure $6 \mathrm{~B}$ ). After $\mathbf{5}_{12}$ treatment, the cell surface became more spongier due to this, which indicated the change in the structure of the cell wall (Figure 6D).
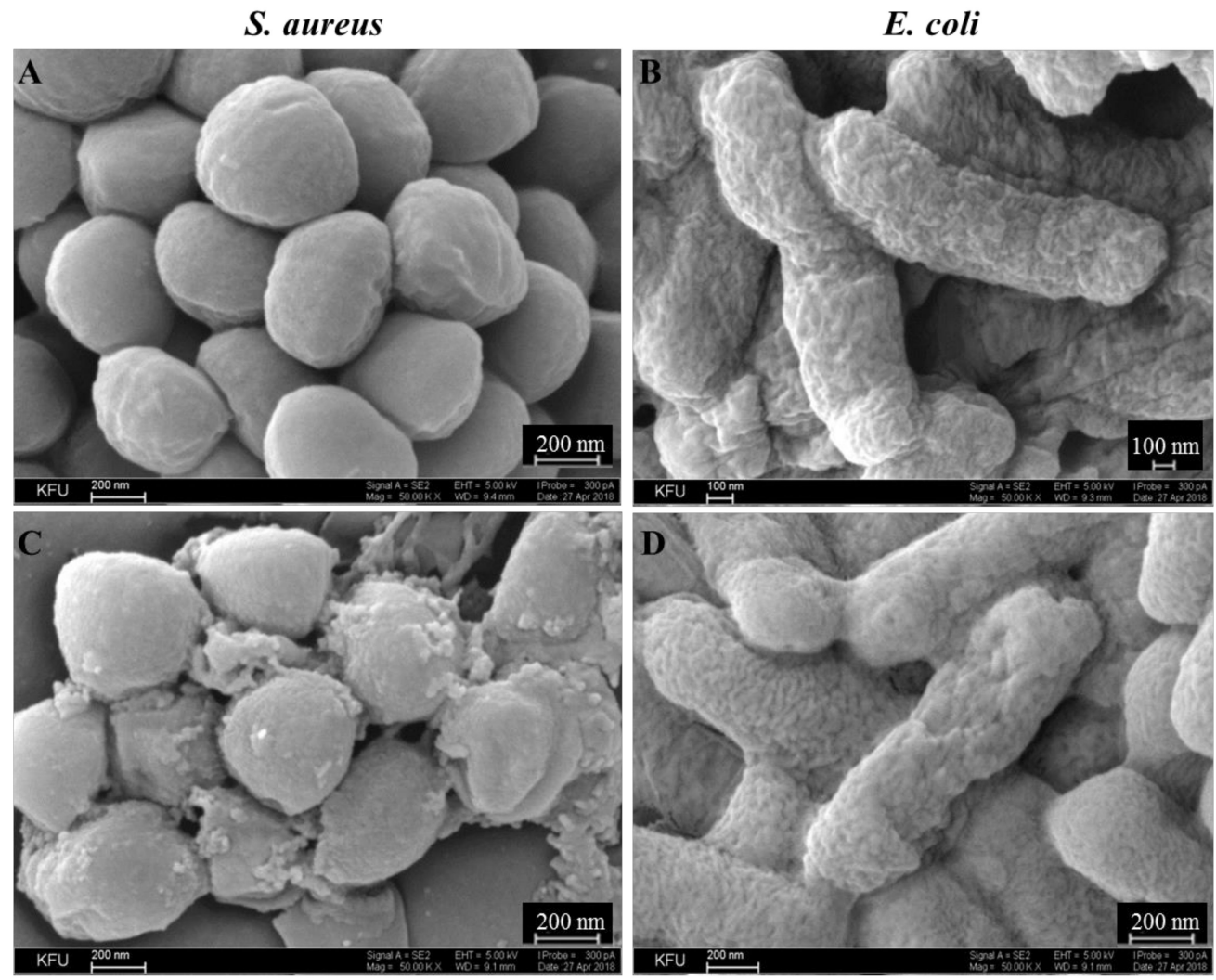

Figure 6. Scanning Electron Microscopy microphotographs of S. aureus ATCC209p (A,C) and E. coli CDC F-50 (B,D). Untreated cells (A,B) and bacteria treated by $0.2 \%$ solution of $\mathbf{5} \mathbf{c}_{\mathbf{1 2}}$ for $30 \mathrm{~min}(\mathbf{C}, \mathbf{D})$. $50,000 \times$ magnification. 


\section{Materials and Methods}

\subsection{Chemistry}

All reagents were obtained from commercial sources and were used without further purification unless otherwise stated. ${ }^{1} \mathrm{H}$ and ${ }^{13} \mathrm{C}$ NMR spectra were recorded on a 'Bruker AVANCE 400 ' at an operating frequency of 400 and $101.56 \mathrm{MHz}$, respectively. Chemical shifts were measured with reference to the residual protons of the solvent $\left(\mathrm{CDCl}_{3},{ }^{1} \mathrm{H}, 7.26 \mathrm{ppm},{ }^{13} \mathrm{C}, 77.16 \mathrm{ppm}, \mathrm{DMSO}-d_{6},{ }^{1} \mathrm{H}\right.$, $\left.2.50 \mathrm{ppm},{ }^{13} \mathrm{C}, 39.51 \mathrm{ppm}\right)$. Coupling constants (J) are given in Hertz (Hz). The following abbreviations are used to describe coupling: $\mathrm{s}=$ singlet, $\mathrm{d}=$ doublet, $\mathrm{t}=$ triplet, $\mathrm{m}=$ multiplet, br $\mathrm{s}=$ broad singlet, br $\mathrm{m}=$ broad multiplet, $\mathrm{AB}=\mathrm{AB}$ system. Melting points were determined using a Stanford Research Systems MPA-100 OptiMelt melting point apparatus and are uncorrected. For TLC (thin-layer chromatography) analysis, silica gel plates from Sorbfil (Krasnodar, Russia) were used with UV light $(254 \mathrm{~nm} / 365 \mathrm{~nm})$ or iron (III) chloride as a developing agent. Column chromatography was performed on silica gel (60-200 mesh) from Acros.

HRMS (high-resolution mass spectrometry) spectra were obtained on a quadrupole time-of-flight ( $t$, qTOF) AB Sciex Triple TOF 5600 mass spectrometer using a turbo-ion spray source (nebulizer gas nitrogen, a positive ionization polarity, needle voltage 5500V). Recording of the spectra was performed in the "TOF MS" mode with collision energy $10 \mathrm{eV}$, declustering potential of $100 \mathrm{eV}$, and a resolution of more than 30,000 full-width half-maximum. Samples with the analyte concentration of $5 \mu \mathrm{mol} / \mathrm{L}$ were prepared by dissolving the test compounds in a mixture of methanol (HPLC-UV Grade, LabScan) and water (LC-MS Grade, Panreac) in a 1:1 ratio.

Methods of synthesis and analytical characteristics of all new synthesized compounds are described in the Electronic Supplementary Material.

\subsection{Antibacterial Activity}

The antibacterial activity of the obtained compounds was evaluated on a number of Gram-positive bacteria: Staphylococcus aureus ATCC 29213, Staphylococcus epidermidis (clinical isolate), Bacillus subtilis 168, and Gram-negative bacteria: Escherichia coli ATCC 25922, Klebsiella pneumoniae 1813 (clinical isolate), Pseudomonas aeruginosa ATCC 27853. The antibacterial activity of compounds $\mathbf{5} \mathbf{a}_{\mathbf{1 2}}, \mathbf{5} \mathbf{c}_{\mathbf{1 0}}, \mathbf{5} \mathbf{c}_{\mathbf{1 2}}, \mathbf{5} \mathbf{d}_{\mathbf{1 0}}, \mathbf{5} \mathbf{f}_{\mathbf{1 0}}$, $5 \mathrm{~g}_{10}, 5 \mathrm{i}_{10}, 5 \mathrm{l}_{10}, \mathbf{5} \mathrm{m}_{8}, \mathbf{5} \mathrm{n}_{10}, \mathbf{5 \mathrm { o } _ { 8 }}, \mathbf{5} \mathrm{p}_{8}$, and $\mathbf{5} \mathrm{q}_{8}$ was additionally evaluated on clinical methicillin-resistant strains of Staphylococcus haemolyticus 837 MRSH (methicillin-resistant Staphylococcus haemolyticus), Staphylococcus aureus 967 MRSA (methicillin-resistant Staphylococcus aureus), Staphylococcus aureus 983 MRSA, Staphylococcus aureus 25, Staphylococcus intermedius 1143 MRSI, Staphylococcus aureus 2020 MRSA, Moraxella sp. 764, Moraxella sp. 765, Moraxella sp. 829, Acinetobacter spp. 1, Acinetobacter spp. 3, Pseudomonas spp. 5, Pseudomonas spp. 6, Klebsiella spp. 11, Klebsiella spp. 12, and Proteus spp. 17, which were obtained from patients with bronchopulmonary diseases in the laboratory of bacteriology of the republic clinical hospital (Kazan, Russia).

The minimum inhibitory concentration (MIC) of compounds was determined by the broth microdilution method in 96-well microtiter plates (Eppendorf), according to the EUCAST (European Committee on Antimicrobial Susceptibility Testing) rules for antimicrobial susceptibility testing with some modifications [16]. The bacterial culture adjusted to $3-9 \times 10^{6}$ cells $/ \mathrm{mL}$ in the Mueller Hinton (MH) broth was seeded into 96-well polystyrol culture plates (Eppendorf). The concentrations of substances to be tested ranged from 0.25 to $64 \mu \mathrm{g} / \mathrm{mL}$ in two-fold serial dilutions. The minimal inhibitory concentration was determined as the lowest concentration of the compound, which leads to no visible bacterial growth after $24 \mathrm{~h}$ of incubation. To determine a minimum bactericidal concentration $(\mathrm{MBC})$, a culture liquid from wells without visible growth was diluted one thousand-fold into new 96-well microtiter plates in fresh $\mathrm{MH}$-broth and incubated for $24 \mathrm{~h}$ growth at $35^{\circ} \mathrm{C}$. MBC was assumed at concentrations where no viable growth could be observed. 


\subsection{Antifungal Activity}

Antifungal activity of compounds $5 \mathrm{a}_{12}, 5 \mathrm{c}_{10}, 5 \mathrm{c}_{12}, 5 \mathrm{~d}_{10}, 5 \mathrm{f}_{10}, 5 \mathrm{~g}_{10}, 5 \mathrm{i}_{10}, 5 \mathbf{l}_{10}, 5 \mathrm{~m}_{8}, 5 \mathrm{n}_{10}, 5 \mathrm{~s}_{8}, 5 \mathrm{p}_{8}$, and $\mathbf{5 q}_{8}$ was evaluated on several fungal strains, which causes cutaneous and systemic mycoses. The fungal isolates were obtained from a collection of the Kazan Institute of Microbiology and Epidemiology (Kazan, Russia): Candida albicans NCTC- 885-653 (etalon strain), Aspergillus niger F-1119 (etalon strain), Trichophyton rubrum 1336 (clinical strain), and Fusarium oxysporum KM-19 (clinical strain). For the inoculum preparation, the pure 2-day and 5-day cultures of yeasts and filamentous fungi, respectively, grown on Sabouraud dense nutrient medium, were used. Yeast cultures of $C$. albicans were prepared by flushing the culture from the surface of the solid agar medium. Cultures of mycelial fungi T. rubrum, F. oxysporum, and A. niger were preground in a mortar. A suspension of microorganisms was prepared in a sterile $0.9 \% \mathrm{NaCl}$ in pure water. The cell concentrations were $(1-5) \times 10^{3}$ for yeast fungi and $(1-5) \times 10^{4}$ for mycelial fungi.

The in vitro evaluation of the antifungal activity of compounds was carried out in a liquid glucose Sabouraud broth in biological test tubes by a two-fold serial dilution approach. Test compounds were prepared at concentrations ranging from 400 to $0.38 \mu \mathrm{g} / \mathrm{mL}$. A test tube in the absence of test compounds served as a control. To each tube, $50 \mu \mathrm{L}$ of inoculum was added. The tubes were incubated for $2-7$ days at $30^{\circ} \mathrm{C}$. To the end of this period, the results were assessed by visual analysis of optical density of the medium. The following MIC endpoints were determined: $0=$ clear solution, no growth, $1=$ weak growth $(25 \%$ control $), 2=$ significant inhibition of growth $(50 \%$ control $), 3=$ insignificant growth inhibition (75\% control), and $4=$ no growth inhibition. All experiments were carried out in duplicate.

\subsection{Cytotoxicity}

The cytotoxicity was evaluated on HEK 293 (human embryonic kidney) cells by using the MTT (3-(4,5-dimethylthiazol-2-yl)-2,5-diphenyltetrazolium bromide) assay. Cells were cultured in $\alpha$-MEM (minimum essential medium) supplemented with 10\% FBS (fetal bovine serum), 2 mM L-glutamine, $100 \mu \mathrm{g} / \mathrm{mL}$ penicillin, and $100 \mu \mathrm{g} / \mathrm{mL}$ streptomycin. Cells were seeded in 96-well plates at the density of 10,000 cells per well and grown overnight at $37^{\circ} \mathrm{C}$ and $5 \% \mathrm{CO}_{2}$ in humidified atmosphere. Then the medium was changed to the fresh one containing compounds to be tested in a concentration of $0.1-100 \mu \mathrm{g} / \mathrm{mL}$. After $72 \mathrm{~h}$ of cultivation, the cultural fluid was discarded and the MTT solution (in DPBS (Dulbecco's phosphate-buffered saline)) was added to the fresh media until reaching a final concentration of $0.5 \mathrm{mg} / \mathrm{mL}$. After $2 \mathrm{~h}$, the liquid was replaced with dimethyl sulfoxide (Sigma-Aldrich, St. Louis, MO) to dissolve formazan crystals, and absorption was measured on Tecan Infinite 200Pro at $550 \mathrm{~nm}$ with a reference of $700 \mathrm{~nm}$. Based on data obtained, the $\mathrm{CC}_{50}$ values (concentrations decreasing the metabolic activity by two-fold) were calculated.

\subsection{Anti-Biofilm Activity}

Bacterial biofilms were grown under static conditions in BM broth $[29,30]$ for $48 \mathrm{~h}$ at $37^{\circ} \mathrm{C}$, washed, and exposed to $1-16 \times$ MBCs of antimicrobials for $24 \mathrm{~h}$ in fresh BM. Then the culture liquid was discarded. The biofilms were washed with sterile $0.9 \% \mathrm{NaCl}$ and suspended in $0.9 \% \mathrm{NaCl}$ by scratching the well bottoms with the following treatment in a sonicator bath for $2 \mathrm{~min}$ at $20 \mathrm{kHz}$ to favor the disintegration of cell clumps, and viable cells were counted using a drop plate assay [31] with modifications [32,33]. Serial 10-fold dilutions of each well were prepared in sterile $0.9 \% \mathrm{NaCl}$ and $5 \mu \mathrm{L}$ of the suspension was dropped onto solid LB (Luria broth) plates. CFUs were counted from those drops containing 5-10 colonies, which were averaged and presented as CFU per $\mathrm{cm}^{2}$.

\subsection{Animals}

Male and female CD-1 (ICR) mice (weighing 18-22 g) were purchased from the Federal Research Center Institute of Cytology and Genetics, Siberian Branch of the Russian Academy of Sciences. 
Male Sprague-Dawley rats weighing 200-230 g were purchased from the laboratory animals nursery "Pushchino," Russia. Animals were given a laboratory rodent diet and water ad libitum. They were housed in polypropylene cages with sawdust at room temperature of $22 \pm 3{ }^{\circ} \mathrm{C}$ and a relative humidity of $50 \pm 20 \%$ with a constant 12-h light/dark cycle. All animals acclimatized for two weeks before the experiment. All experimental procedures were performed in accordance with the Ethical Principles in Animal Research and were approved by the Local Ethics Committee №2-2017 of the Kazan Federal University.

\subsection{In Vivo Toxicity}

Mice were stratified by weight and randomly assigned into one sex group of three per group (for initiating dose). The number of animals in the group was increased to six per group if necessary. Prior to the experiment, animals were weighed, marked, and fasted for $8 \mathrm{~h}$ with free access to water. Compounds $\mathbf{5} \mathbf{c}_{\mathbf{1 2}}, \mathbf{5} \mathbf{d}_{\mathbf{1 0}}$, and $\mathbf{5} \mathbf{p}_{\mathbf{8}}$ were dissolved in distilled water and administered to the mice by oral gavage at doses 50, 100, 500, 1000, 1500, and $2000 \mathrm{mg} / \mathrm{kg}$ body weight. The initial dose of $1000 \mathrm{mg} / \mathrm{kg}$ was selected as the dose expected to produce mortality in some animals. Further groups of animals have been dosed at higher or lower fixed doses, depending on the presence of mortality, until the study objective was achieved. All animals were observed twice daily for symptoms and mortality for two weeks. All surviving animals were euthanized with $\mathrm{CO}_{2}$ inhalation at the end of the study on day 14, and their vital organs were individually observed for overt pathology by necropsy, and the $\mathrm{LD}_{50}$ was calculated by Probit analysis in IBM SPSS Statistics software.

\subsection{In Vivo Efficiency in a Rat Skin Model}

Gram-negative (E. coli CDC F-50 ${ }^{\mathrm{TM}}$ ) bacteria were used as test organisms for the in vivo rat skin model. The culture of E. coli CDC F-50, grown on Mueller-Hinton's medium (MHB, NICF, Russia) for 18-20 h, were washed off with sterile isotonic chloride solution, and centrifuged at $5000 \mathrm{rpm}$ for $5 \mathrm{~min}$. The supernatant was discharged and the cells were resuspended with sterile isotonic sodium chloride solution.

Endo medium (HiMedia Laboratories Pvt. Ltd., Mumbai, India) were used according to the manufacturers' instructions. Phosphate-buffered saline (PBS, Ekoservice, Russia), Tween 80 (Sigma-Aldrich, St Louis, MO, USA), saponin (Dia-M, Moscow, Russia), histidine (Sigma-Aldrich, St Louis, MO, USA), and cysteine (Sigma-Aldrich, St Louis, MO, USA) were used for neutralizing solution ( $3 \% v / v$ Tween $80,3 \% w / v$ saponin, $0.1 \% w / v$ histidine, $0.1 \% w / v$ cysteine, PBS).

Thirty rats were randomly assigned to five groups: four experimental groups and one control group with six rats in each group. All rats were anesthetized with isoflurane (induction $3.0 \%$ in air, maintenance of $1.5 \%$ in air) [34]. On the experimental day, dorsal sites of the animals more than $5 \times 5 \mathrm{~cm}$ were shaved, and squares $5 \times 5 \mathrm{~cm}$ were outlined with the marker. Suspension $(0.25 \mathrm{~mL})$ containing $1 \times 10^{7}$ CFU E. coli CDC F-50 were applied on the selected area, distributed with a sterile disposable spatula over the entire area of the square, and left for 3-4 min until it dries. Then a gauze cloth $(5 \times 5 \mathrm{~cm})$ was applied to the rat's skin for $5 \mathrm{~min}$. In experimental groups, gauze cloths were preliminarily wetted in $1 \mathrm{~mL}$ of $0.2 \% 5 \mathbf{c}_{12}$, miramistin, benzalkonium chloride or chlorhexidine solutions. In a negative control group, the tissue was immersed in $1 \mathrm{~mL}$ of sterile saline. At the end of the exposure time in experimental groups, the neutralizer was used to stop antiseptic action on bacteria. The rat's skin was washed with a sterile gauze $(5 \times 5 \mathrm{~cm})$ moistened with a sterile neutralizer solution. Then, the gauze cloth was transferred into $50-\mathrm{mL}$ tubes with sterile physiological saline $(10 \mathrm{~mL})$ and shaken for $10 \mathrm{~min}$. Then the washing liquid was placed into two to three plates $(0.1 \mathrm{~mL}$ on each plate) in a selective Endo medium, and cultured for $24-48 \mathrm{~h}$ at $37^{\circ} \mathrm{C}$.

After the time required for cultivation of microorganisms, the results were recorded by the number of colonies grown on the Petri dish. The results were taken into account by assessing the residual contamination of surfaces after treating them with antiseptic solution. After counting the number of colonies grown on Petri dishes, the density of contamination per $1 \mathrm{~cm}^{2}$ of the surface and the 
percentage of disinfection were calculated, which corresponds to the number of colonies removed from the control surfaces that were not exposed to antiseptics as 100\%. The percentage of growth inhibition of E. coli CDC F-50 was calculated using the following formula:

$$
\mathrm{I}=100 \%-\frac{\mathrm{E} \times 100 \%}{\mathrm{C}}, \text { where }
$$

I-percentage of growth inhibition, $\%$,

$\mathrm{C}$ - the number of colonies in the control group,

$\mathrm{E}$ - the number of colonies in the experimental group.

\subsection{Outer Membrane Permeability}

The outer membrane permeability was assessed by measuring the fluorescent dye NPN (1-N-phenylnaphthylamine) uptake [35,36]. Gram-negative bacteria (E. coli CDCF-50) were incubated to mid-log phase in Mueller Hinton broth $\left(37{ }^{\circ} \mathrm{C}, 120 \mathrm{rpm}\right)$, harvested by centrifugation (3.000 rpm for $10 \mathrm{~min}$ ), washed twice, and diluted to $10^{5} \mathrm{CFU} / \mathrm{mL}$ with $5 \mathrm{mM}$ HEPES (2-[4-(2-hydroxyethyl)piperazin-1-yl]ethanesulfonic acid) buffer ( $\mathrm{pH} 7.4$, containing $5 \mathrm{mM}$ glucose). Then, $200 \mu \mathrm{L}$ of suspension were placed into a 96-well plate which were mixed with $10 \mu \mathrm{M}$ of NPN. The background fluorescence was recorded (excitation $\lambda=350 \mathrm{~nm}$, emission $\lambda=420 \mathrm{~nm}$ ) by Spectrophotometer Infinite M200 PRO (Tecan, Switzerland). Next, $20 \mu \mathrm{L}$ of $\mathbf{5} \mathbf{c}_{12}$ with final concentrations equal to minimal bactericidal concentration $(\mathrm{MBC}), 2 \times \mathrm{MBC}$, and $4 \times \mathrm{MBC}$, was added to the E. coli suspension into a 96-well plate. The fluorescence was recorded until no further increase of fluorescence was detected. The NPN uptake percent was calculated using the following formula:

$$
\text { NPN uptake }(\%)=(\text { Fobs }- \text { F0) } /(\text { F100 }- \text { F0 }) \times 100 \% \text {, where }
$$

Fobs-fluorescence of $\mathbf{5} \mathbf{c}_{12}$ in $\mathrm{MBC} / 2 \times \mathrm{MBC} / 4 \times \mathrm{MBC}$,

F0-the initial NPN fluorescence with $E$. coli in the absence of $\mathbf{5} \mathbf{c}_{\mathbf{1 2}}$,

F100—NPN fluorescence with E. coli after addition of $10 \mu \mathrm{g} / \mathrm{mL}$ polymyxin B (Sigma-Aldrich, St Louis, MO, USA), which was used as a positive control.

\subsection{Cytoplasmic Membrane Depolarization Assays}

The cytoplasmic membrane depolarization was determined by using the membrane potential-sensitive fluorescent dye 3,3-Dipropylthiadicarbocyanine Iodide $\mathrm{DiSC}_{3}$ (5), (Sigma-Aldrich). Gram-positive (S. aureus ATCC209p) and Gram-negative (E. coli CDCF-50) bacteria were incubated to a mid-log phase in Mueller Hinton broth $\left(37^{\circ} \mathrm{C}, 120 \mathrm{rpm}\right)$, harvested by centrifugation $(3.000 \mathrm{rpm}$ for $10 \mathrm{~min}$ ), washed twice, and diluted to an optical density of 0.05 at $600 \mathrm{~nm}$ with $5 \mathrm{mM}$ HEPES buffer ( $\mathrm{pH} 7.4$, containing $20 \mathrm{mM}$ glucose) containing $0.1 \mathrm{M} \mathrm{KCl}$ to equilibrate the cytoplasmic and external $\mathrm{K}^{+}$. Then, the cell suspensions $(180 \mu \mathrm{L})$ were mixed with $20 \mu \mathrm{L}$ of $0.8 \mu \mathrm{M} \mathrm{DiSC}_{3}(5)$ in a 96 -well plate and incubated for $15 \mathrm{~min}$ in darkness (until maximal dye uptake was reached, the background fluorescence was recorded (excitation $\lambda=620 \mathrm{~nm}$, emission $\lambda=670 \mathrm{~nm}$ ) by Spectrophotometer Infinite M200 PRO (Tecan, Switzerland). After that, $\mathbf{5} \mathbf{c}_{\mathbf{1 2}}$ and reference compounds (benzalkonium chloride, miramistin) in $0.5,1$, and $2 \times$ MBCs were added to cell suspensions into a 96-well plate. Changes in fluorescence were observed during $20 \mathrm{~min}$ of recording.

\subsection{Scanning Electron Microscopy}

The analysis was carried out using a high-resolution scanning electron microscope Merlin (Carl Zeiss, oberkochen, Germany). The surface morphology was studied at an accelerating voltage of primary electrons of $5 \mathrm{kV}$ and a probe current of $300 \mathrm{pA}$ for a minimal impact on the study object. Scanning electron microscopy was carried out in the Interdisciplinary center for Analytical Microscopy (Kazan Federal University). 


\section{Conclusions}

In conclusion, an extended set of 108 novel quaternary bis-ammonium pyridoxine derivatives carrying various substituents at the quaternary nitrogens and acetal carbon have been synthesized. In the course of primary in vitro screening 13 compounds $\left(\mathbf{5 a}_{\mathbf{1 2}}, \mathbf{5} \mathbf{c}_{\mathbf{1 0}}, \mathbf{5} \mathbf{c}_{\mathbf{1 2}}, \mathbf{5} \mathbf{d}_{\mathbf{1 0}}, \mathbf{5} \mathbf{f}_{\mathbf{1 0}}, \mathbf{5} \mathbf{g}_{\mathbf{1 0}}, \mathbf{5} \mathbf{i}_{\mathbf{1 0}}, \mathbf{5} \mathbf{1}_{\mathbf{1 0}}, \mathbf{5} \mathbf{m}_{\mathbf{8}}\right.$, $5 \mathbf{n}_{10}, 5 \mathbf{o}_{8}, 5 \mathbf{p}_{8}$, and $\mathbf{5} \mathbf{q}_{\mathbf{8}}$ ) exhibiting comparable or superior antibacterial activity compared to miramistin, benzalkonium chloride and chlorhexidine were identified (MICs $0.25-4 \mu \mathrm{g} / \mathrm{mL}$ on Gram-positive and $0.5-16 \mu \mathrm{g} / \mathrm{mL}$ on Gram-negative bacteria). A strong correlation between the lipophilicity and antimicrobial activity was found. Most active compounds had $\log P$ values in the range of $1-3$, while compounds with $\log \mathrm{P}>6$ and $\log \mathrm{P}<0$ were almost inactive. In further investigation of antibacterial and antifungal activity on clinical bacterial and fungal isolates, compounds $\mathbf{5} \mathbf{c}_{\mathbf{1 2}}, \mathbf{5} \mathbf{i}_{\mathbf{1 0}}, \mathbf{5} \mathbf{1}_{\mathbf{1 0}}$, $5 \mathbf{n}_{10}, 5 \mathbf{o}_{8}, 5 p_{8}$, and $\mathbf{5} q_{8}$ significantly exceeded miramistin, benzalkonium chloride and chlorhexidine in antibacterial activity. Compounds $\mathbf{5} \mathbf{c}_{\mathbf{1 0}}, \mathbf{5} \mathbf{c}_{\mathbf{1 2}}, \mathbf{5} \mathbf{d}_{\mathbf{1 0}}, \mathbf{5} \mathbf{f}_{\mathbf{1 0}}, \mathbf{5} \mathbf{n}_{\mathbf{1 0}}$, and $\mathbf{5} \mathbf{p}_{\mathbf{8}}$ exhibited a pronounced antimycotic activity than all reference antiseptics against all fungi tested. All active compounds had comparable values of cytotoxicity with miramistin and chlorhexidine and were less toxic when compared to benzalkonium chloride. In vivo, the leading compound $\mathbf{5 c}_{12}$ was considerably less toxic $\left(\mathrm{LD}_{50} 1705 \mathrm{mg} / \mathrm{kg}\right)$ than benzalkonium chloride $\left(\mathrm{LD}_{50} 180 \mathrm{mg} / \mathrm{kg}\right)$, miramistine ( $\mathrm{LD}_{50} 1000 \mathrm{mg} / \mathrm{kg}$ ) and chlorhexidine ( $\left(\mathrm{LD}_{50} 1260 \mathrm{mg} / \mathrm{kg}\right.$ ) at oral administration on CD-1 mice. Moreover, $\mathbf{5} \mathbf{c}_{12}$ demonstrated comparable or even higher activity than that of the benzalkonium chloride on biofilm-embedded S. aureus, S. epidermidis, E. coli, and P. aeruginosa. A $0.2 \%$ aqueous solution of $\mathbf{5} \mathbf{c}_{\mathbf{1 2}}$ showed $97.5 \%$ growth inhibition of E. coli CD CF-50 after 5 min of exposure on rat's skin comparable to reference drugs efficiency. The molecular mechanism of antibacterial activity of $\mathbf{5 c}_{12}$ apparently is the membrane damage since a $\mathbf{5} \mathbf{c}_{12}$ dose-dependent effect on membrane permeability and polarization was observed. The obtained results allow suggesting described quaternary bis-ammonium pyridoxine derivatives as promising lead molecules for the development of the new antiseptics with a broad spectrum of antimicrobial and antifungal activity.

Supplementary Materials: The following are available online at http://www.mdpi.com/1420-3049/25/18/4341/s1, methods of synthesis, analytical characteristics, and copies of ${ }^{1} \mathrm{H}$ and ${ }^{13} \mathrm{C}$ NMR spectra of all new synthesized compounds are described in the Electronic Supplementary Material.

Author Contributions: N.V.S. performed the synthesis of compounds, analyzed results and wrote this paper. M.V.P., S.V.S., M.R.G., R.M.V. and R.S.P. performed the synthesis of compounds and wrote the Supplementary Material. R.R.K., M.N.A. and A.R.K. performed the biological experiments and wrote this paper. D.Y.G. performed the mass-spectra shooting and analysis. A.G.I., E.S.K., E.V.N., S.A.L., M.I.Z. and A.E.S. performed the biological experiments. Y.G.S. conceived and supervised the project, analyzed results, and wrote this paper. All authors have read and agreed to the published version of the manuscript.

Funding: This work was supported by subsidy allocated to Kazan Federal University for the state assignment in the sphere of scientific activities (project number 0671-2020-0053).

Conflicts of Interest: The authors declare no conflict of interest. The funders had no role in the design of the study, in the collection, analyses, or interpretation of data, in the writing of the manuscript, or in the decision to publish the results.

\section{References}

1. Review on Antimicrobial Resistance. Antimicrobial Resistance: Tackling a Crisis for the Health and Wealth of Nations; Review on AMR, Wellcome Trust, HM Government: London, UK, 2014. Available online: http://www.jpiamr.eu/wp-content/uploads/2014/12/AMR-Review-Paper-Tackling-a-crisisfor-the-health-and-wealth-of-nations_1-2.pdf (accessed on 20 July 2020).

2. Frieri, M.; Kumar, K.; Boutin, A. Antibiotic resistance. J. Infect. Public Health. 2017, 10, 369-378. [CrossRef] [PubMed]

3. McDonnell, G.; Russell, A.D. Antiseptics and disinfectants: Activity, action, and resistance. Clin. Microbiol. Rev. 1999, 12, 147-179. [CrossRef] [PubMed]

4. Sheldon, A.T., Jr. Antiseptic "resistance": Real or perceived threat? Clin. Infect. Dis. 2005, 40, 1650-1656. [CrossRef] [PubMed] 
5. Roberts, C.D.; Leaper, D.J.; Assadian, O. The role of topical antiseptic agents within antimicrobial stewardship strategies for prevention and treatment of surgical site and chronic open wound infection. Adv. Wound Care 2017, 6, 63-71. [CrossRef]

6. Gilbert, P.; Moore, L.E. Cationic antiseptics: Diversity of action under a common epithet. J. Appl. Microbiol. 2005, 99, 703-715. [CrossRef]

7. Osmanov, A.; Farooq, Z.; Richardson, M.D.; Denning, D.W. The antiseptic Miramistin: A review of its comparative in vitro and clinical activity. FEMS Microbiol. Rev. 2020, 44, 399-417. [CrossRef]

8. Vijayakumar, R.; Sandle, T. A review on biocide reduced susceptibility due to plasmid-borne antiseptic-resistant genes-special notes on pharmaceutical environmental isolates. J. Appl. Microbiol. 2019, 126, 1011-1022. [CrossRef]

9. Pereira, B.M.P.; Tagkopoulos, I. Benzalkonium chlorides: Uses, regulatory status, and microbial resistance. Appl. Environ. Microbiol. 2019, 85, e00377-19.

10. Kano, S.; Sugibayashi, K. Kinetic analysis on the skin disposition of cytotoxicity as an index of skin irritation produced by cetylpyridinium chloride: Comparison of in vitro data using a three-dimensional cultured human skin model with in vivo results in hairless mice. Pharm. Res. 2006, 23, 329-335. [CrossRef]

11. Jennings, M.C.; Minbiole, K.P.C.; Wuest, W.M. Quaternary ammonium compounds: An antimicrobial mainstay and platform for innovation to address bacterial resistance. ACS Infect. Dis. 2015, 1, 288-303. [CrossRef]

12. Shtyrlin, Y.G.; Petukhov, A.S.; Strelnik, A.D.; Shtyrlin, N.V.; Iksanova, A.G.; Pugachev, M.V.; Pavelyev, R.S.; Dzyurkevich, M.S.; Garipov, M.R.; Balakin, K.V. Chemistry of pyridoxine in drug design. Russ. Chem. Bull. 2019, 68, 911-945. [CrossRef]

13. Shtyrlin, N.V.; Sapozhnikov, S.V.; Koshkin, S.A.; Iksanova, A.G.; Sabirov, A.H.; Kayumov, A.R.; Nureeva, A.A.; Zeldi, M.I.; Shtyrlin, Y.G. Synthesis and antibacterial activity of novel quaternary ammonium pyridoxine derivatives. Med. Chem. 2015, 11, 656-665. [CrossRef]

14. Sapozhnikov, S.V.; Shtyrlin, N.V.; Kayumov, A.R.; Zamaldinova, A.E.; Iksanova, A.G.; Nikitina, E.V.; Krylova, E.S.; Grishaev, D.Y.; Balakin, K.V.; Shtyrlin, Y.G. New quaternary ammonium pyridoxine derivatives: Synthesis and antibacterial activity. Med. Chem. Res. 2017, 26, 3188-3202. [CrossRef]

15. Shtyrlin, N.V.; Sapozhnikov, S.V.; Galiullina, A.S.; Kayumov, A.R.; Bondar, O.V.; Mirchink, E.P.; Isakova, E.B.; Firsov, A.A.; Balakin, K.V. Synthesis and antibacterial activity of quaternary ammonium 4-deoxypyridoxine derivatives. Biomed. Res. Int. 2016, 2016, 3864193. [CrossRef] [PubMed]

16. Nikitina, E.V.; Zeldi, M.I.; Pugachev, M.V.; Sapozhnikov, S.V.; Shtyrlin, N.V.; Kuznetsova, S.V.; Evtygin, V.E.; Bogachev, M.I.; Kayumov, A.R.; Shtyrlin, Y.G. Antibacterial effects of quaternary bis-phosphonium and ammonium salts of pyridoxine on Staphylococcus aureus cells: A single base hitting two distinct targets? World J. Microbiol. Biotechnol. 2016, 32, 1-7. [CrossRef] [PubMed]

17. Shtyrlin, Y.G.; Shtyrlin, N.V.; Sapozhnikov, S.V.; Iksanova, A.G.; Kazakova, R.R.; Agafonova, M.N.; Strelnik, A.D. Antiseptic drug. Patent RU No. 2641309, 17 January 2018.

18. Shtyrlin, Y.G.; Shtyrlin, N.V.; Iksanova, A.G.; Nikitina, E.V.; Kazakova, R.R.; Lisovskaya, S.A. Quaternary Ammonium Salt Possessing Antimicrobial and Antibacterial Activity. Patent RU No. 2,666,544 C1, 11 September 2018.

19. Shtyrlin, Y.G.; Shtyrlin, N.V.; Sapozhnikov, S.V.; Pavelyev, R.S. Method of producing 5,8-(bis(methylene(N,Ndimethyl-N-dodecylammonium))-2-ethyl-4H-[1,3]dioxino[4,5-c]pyridinium dichloride. Application RU No. 2,697,848 C1, 21 August 2019.

20. Grigor'ev, A.A.; Shtyrlin, N.V.; Gabbasova, R.R.; Zeldi, M.I.; Grishaev, D.Y.; Gnezdilov, O.I.; Balakin, K.V.; Nasakin, O.E.; Shtyrlin, Y.G. Synthesis, antibacterial and antitumor activity of methylpyridinium salts of pyridoxine functionalized 2-amino-6-sulfanylpyridine-3,5-dicarbonitriles. Synth. Comm. 2018, 48, 2288-2304.

21. Pavelyev, R.S.; Bondar, O.V.; Nguyen, T.N.T.; Ziganshina, A.A.; Al Farroukh, M.; Karwt, R.; Alekbaeva, G.D.; Pugachev, M.V.; Yamaleeva, Z.R.; Kataeva, O.N.; et al. Synthesis and in vitro antitumor activity of novel alkenyl derivatives of pyridoxine, bioisosteric analogs of feruloyl methane. Bioorg. Med. Chem. 2018, 26, 5824-5837. [CrossRef]

22. Csizmadia, F.; Tsantili-Kakoulidou, A.; Panderi, I.; Darvas, F. Prediction of distribution coefficient from structure. 1. Estimation method. J. Pharm. Sci. 1997, 86, 865-871. [CrossRef]

23. United Nations. Globally Harmonized System of Classification and Labeling of Chemicals (GHS), 4th ed.; United Nations: New York, NY, USA; Geneva, Switzerland, 2011; pp. 109-120. 
24. Bodor, N. Labile quaternary ammonium salts as soft antimicrobials. J. Med. Chem. 1980, 23, 469-474. [CrossRef]

25. Krivoshein, J.S.; Rud'ko, A.P. Therapeutic Preparation. Patent RU No. 2,161,961, 20 January 2001.

26. Material Safety Data Sheet. Available online: https://pfe-pfizercom-d8-prod.s3.amazonaws.com/products/ material_safety_data/PZ00711.pdf (accessed on 1 December 2007).

27. Agafonova, M.N.; Kazakova, R.R.; Lubina, A.P.; Zeldi, M.I.; Nikitina, E.V.; Balakin, K.V.; Shtyrlin, Y.G. Antibacterial activity profile of miramistin in in vitro and in vivo models. Microb. Pathog. 2020, 142, 104072. [CrossRef]

28. Merianos, J.J. Surface-active agents. In Block's Disinfection, Sterilization, and Preservation; Lippincott Williams \& Wilkins: Philadelphia, PA, USA, 2001; pp. 63-320.

29. Kayumov, A.R.; Khakimullina, E.N.; Sharafutdinov, I.S.; Trizna, E.Y.; Latypova, L.Z.; Lien, H.T.; Margulis, A.B.; Bogachev, M.I.; Kurbangalieva, A.R. Inhibition of biofilm formation in Bacillus subtilis by new halogenated furanones. J. Antibiot. 2015, 68, 297-301. [CrossRef] [PubMed]

30. Trizna, E.Y.; Khakimullina, E.N.; Latypova, L.Z.; Kurbangalieva, A.R.; Sharafutdinov, I.S.; Evtyugin, V.G.; Babynin, E.V.; Bogachev, M.I.; Kayumov, A.R. Thio derivatives of 2(5H)-furanone as inhibitors against bacillus subtilis biofilms. Acta Nat. 2015, 7, 102-107. [CrossRef]

31. Herigstad, B.; Hamilton, M.; Heersink, J. How to optimize the drop plate method for enumerating bacteria. J. Microbiol. Methods 2001, 44, 121-129. [CrossRef]

32. Sharafutdinov, I.S.; Trizna, E.Y.; Baidamshina, D.R.; Ryzhikova, M.N.; Sibgatullina, R.R.; Khabibrakhmanova, A.M.; Latypova, L.Z.; Kurbangalieva, A.R.; Rozhina, E.V.; Klinger-Strobel, M.; et al. Antimicrobial effects of sulfonyl derivative of $2(5 \mathrm{H})$-furanone against planktonic and biofilm associated methicillin-resistant and -susceptible Staphylococcus aureus. Front. Microbiol. 2017, 8, 2246. [CrossRef] [PubMed]

33. Baidamshina, D.R.; Trizna, E.Y.; Holyavka, M.G.; Bogachev, M.I.; Artyukhov, V.G.; Akhatova, F.S.; Rozhina, E.V.; Fakhrullin, R.F.; Kayumov, A.R. Targeting microbial biofilms using Ficin, a nonspecific plant protease. Sci. Rep. 2017, 7, 46068. [CrossRef] [PubMed]

34. Kazakova, R.R.; Luong, D.T. Anesthetics for magnetic resonance studies in rodents (Systematic review of experimental results). Eksp. Klin. Farmakol. 2016, 79, 39-44.

35. Wang, J.; Chou, S.; Xu, L.; Zhu, X.; Dong, N.; Shan, A.; Chen, Z. High specific selectivity and Membrane-Active Mechanism of the synthetic centrosymmetric $\alpha$-helical peptides with Gly-Gly pairs. Sci. Rep. 2015, 5, 15963. [CrossRef]

36. Zhu, X.; Dong, N.; Wang, Z.; Ma, Z.; Zhang, L.; Ma, Q.; Shan, A. Design of imperfectly amphipathic a-helical antimicrobial peptides with enhanced cell selectivity. Acta Biomater. 2014, 10, 244-257. [CrossRef]

Sample Availability: Samples of the compounds 5a8-5r18 are available from the authors.

(C) 2020 by the authors. Licensee MDPI, Basel, Switzerland. This article is an open access article distributed under the terms and conditions of the Creative Commons Attribution (CC BY) license (http://creativecommons.org/licenses/by/4.0/). 